\title{
1 Integrative functional analysis uncovers metabolic differences between Candida species
} 2

3 Neelu Begum ${ }^{1}$, Sunjae Lee ${ }^{1}$, Aize Pellon ${ }^{1}$, Shervin Sadeghi Nasab ${ }^{1}$, Jens Nieslen ${ }^{2,3}$, Mathias

4 Uhlen $^{4}$, David Moyes ${ }^{1 *}$, Saeed Shoaie ${ }^{1,4^{*}}$

5

$6{ }^{1}$ Centre for Host-Microbiome Interactions, Faculty of Dentistry, Oral \& Craniofacial

7 Sciences, King's College London, SE1 9RT, UK.

$8{ }^{2}$ Department of Biology and Biological Engineering, Kemivägen 10, Chalmers University

9 of Technology, SE-412 96, Gothenburg, Sweden.

$10{ }^{3}$ BioInnovation Institute, Ole Maaløes Vej 3, DK2200 Copenhagen N, Denmark

$11{ }^{4}$ Science for Life Laboratory, KTH - Royal Institute of Technology, Stockholm, SE-171 21, 12 Sweden.

$13 *$ corresponded to: david.moyes@kcl.ac.uk; saeed.shoaie@kcl.ac.uk 


\section{Abstract}

17 Candida species are a dominant constituent of the human mycobiome and a better

18 understanding of their metabolism from a fungal perspective can provide key insights into

19 their ability to cause pathogenesis. Here, we have developed the BioFung database - a fungal

20 specific tool for functional annotation using the KEGG database that provides an efficient

21 method for annotation of protein-encoding gene. Analysis of carbohydrate-active enzyme

22 (CAZymes) and BioFung, uncovered core and accessory features across Candida species

23 demonstrating plasticity, adaptation to the environment and acquired features. Integerative

24 functional analysis revealed that all Candida species can employ amino acid metabolism.

25 However, metabolomics revealed that only a specific cluster of species (AGAu species - C.

26 albicans, C. glabrata and C. auris) utilised amino acid metabolism. We identified critical

27 metabolic pathways in the AGAu clusters with biomarkers and anti-fungal target potential in

28 the CAZyme profile, polyamine, choline and fatty acid biosynthesis pathways. This study,

29 combining genomic analysis, metabolomics and gene expression validation, highlights the

30 metabolic diversity within AGAu species that underlies their remarkable ability to dominate

31 the mycobiome and cause disease. 


\section{Introduction}

$34 \quad$ Fungal infections affect around 7.5 million people around the world every year.

35 Within human fungal communities (mycobiome), with the notable exception of the skin,

36 Candida species are the most common group ${ }^{1-3}$. These species are generally pathobionts,

37 being the most common human fungal pathogens, despite also being commensal organisms ${ }^{4}$.

38 Candida infections are becoming increasingly concerning, and the World Health

39 Organisation (WHO) has recently emphasised international surveillance for diagnosis and 40 management of fungal infection, particularly C. albicans infection ${ }^{5-7}$. Recently, a novel

41 Candida species, Candida auris has been identified with significant mortality and morbidity, 42 as well as a high degree of antifungal resistance ${ }^{8,9}$. There are over 200 Candida species 43 currently identified, but only a handful of these are present in the human microbiota with the 44 ability to cause infection and pathology. Most notable examples of these include C. albicans, 45 C. glabrata, C. dubliniensis, C. tropicalis and C. auris ${ }^{9-15}$. Candida species, most notably C. 46 albicans and C. glabrata, can give rise to a variety of superficial infections, including oral 47 thrush and vulvovaginal candidiasis, but are also capable of causing a systemic infection with 48 significant mortality ${ }^{16-20}$. As well as direct infections, fungi such as Candida species have 49 also been associated with oncogenesis through complement activation, demonstrating 50 potential effects of the interaction of fungal species with human host ${ }^{21}$.

51

52 An essential virulence determinant of fungi is their metabolic plasticity ${ }^{22}$. Fungal are 53 significant in their ability to utilise numerous different anabolic and catabolic sources in their 54 metabolic processes, attributable to switching between carbon and nitrogen sources ${ }^{23}$. 55 Nutritional availability, environmental factors, competition and pathogenic factors all 56 influence this plasticity ${ }^{24,25}$. Investigations of Candida species-specific transcriptional 57 regulators of glycolytic genes (e.g. Tye2 and Gal4) and enzymes of the glycolytic pathway 58 (hexose catabolism), indicate these factors play an essential role in central carbon metabolism 59 commonly applied during infection events ${ }^{22,24,26}$. Glycolytic metabolism can activate 60 virulence factors that initiate hypha formation, activate fermentative pathways, repress 61 gluconeogenesis, and the TCA $\operatorname{cycle}^{27-30}$. Alternatively, C. albicans can switch to 62 gluconeogenesis and the glyoxylate cycle to confer full pathogenesis during systemic 63 candidiasis $^{31-34}$. Carbohydrate metabolism is coupled with changes of cell wall architecture, 64 host immune response modulation, as well as adherence, biofilm formation, stress response 65 and drug resistance ${ }^{24,35-37}$. If carbohydrate sources are limited, Candida species can use 66 amino acids and lipids as supplementation for metabolic adaptation ${ }^{22,38-40}$. Amino acids 
67 produced by $C$. albicans have been shown to drive tissue damage by initiating stress 68 responses and adjusting the surrounding environmental $\mathrm{pH}$, helping induce of host invasion 69 processes ${ }^{35,41-47}$. Very little is known about the regulation, process and utilisation of amino 70 acid metabolism in Candida ${ }^{39}$. However, C. albicans is known to use amino acids to replace 71 carbon and other nitrogen sources ${ }^{48}$. Candida's ability to convert arginine to urea allows the 72 neutralisation of an acidic environment triggering the development of hyphae and biofilm

73 formation $32,49,50$. Notably, recent work has shown that C. albicans phagocyted by 74 macrophages induces fatty acid $\beta$-oxidation and the glyoxylate pathway to induce hypha 75 formation for escape. In a harsh environment that lacks even a nitrogen source, Candida can 76 recycle and produce its own proteins and polyamines without host nitrate ${ }^{51}$. Thus, 77 understanding of metabolism and functionality of Candida is instrumental in tackling 78 infection and mortality prevalence ${ }^{52}$.

80 Here we developed BioFung - a database focused on functional information and 81 interpretation of biological information. It is based on 128 fungal species using KEGG 82 orthologs (KO). By applying BioFung to Candida species, we identified a distinct cluster of 83 C. albicans, C. glabrata and C. auris referred to as AGAu species. This cluster has a high 84 association with infection and mortality, relative to other Candida species ${ }^{18,53-55}$. We applied comparative analysis techniques based on gene, protein, and enzyme-substrate levels and identified metabolic pathways in Candida species, such as choline and polyamine pathways. Metabolomics along with experimental validation from gene expression confirmed the AGAu cluster difference. This study (1) provides a novel tool for functional annotation of fungal species, (2) highlights amino acid metabolism importance in AGAu species, and (3) identifies novel potential fungal biomarkers and anti-fungal targets in metabolic pathway.

\section{Results}

Development of BioFung database and functional annotation of Candida proteinencoding genes

To perform a global functional analysis of Candida species, we collected 49 publicly available genomes of different Candida strains covering 13 different species (Supplementary Table 1). We selected species based on their clinical importance and abundance within the human mycobiome ${ }^{56-60}$. All 49 Candida strains were isolated from different body sites from people in different geographical locations (Fig. 1a and Extended Fig. 1a). The quality of the 
101 genome of each strain was checked at the level of scaffold assembly and genome size

102 (Extended Fig. 1b before reassessing the phylogenetic relationship of these 49 strains based 103 on nucleotide sequence using average nucleotide identity (ANI) (Fig. 1b, Method). We 104 observed strain-specific differences in phylogenetic lineages with 11 distinct branches, 105 including branching of C. auris, C. glabrata and C. albicans, implying genetic diversity that 106 could implicitly be interpreted into functional variances. To elucidate functional details for 107 these strains, we built fungi-specific Hidden Markov Models (HMM) using fungal gene 108 clusters, named BioFung database (Fig. 1c, Method) ${ }^{61,62}$. We analysed 524,288 fungal genes, 109 from 128 fungal species, with a coverage of 4,822 KOs, and 4,430 fungal $\mathrm{KO}$ alignments to 110 create BioFung (Supplementary Table 2-4). Comparison of the BioFung database with other 111 eukaryote-specific HMM sources indicated that the BioFung has both higher coverage and 112 specificity of KOs (Extended fig. 1c-e) ${ }^{61,62}$.

114 The collection of Candida strains used to integrate functional annotations can be categorised 115 into commonly invasive, non-invasive (requiring a secondary factor to cause infection, such 116 as co-morbidity, immunodeficiency) based on literature (Fig. 1d, Supplementary Table 5, 117 Method). These representative samples of Candida were integrated into the functional 118 analysis framework, with a total of 49 Candida species annotated with BioFung, Protein 119 families (Pfam) ${ }^{63}$ and Carbohydrate-Active enZyme (CAZyme) ${ }^{64}$ databases. We applied 120 BioFung using the UCLUST algorithm, to establish core genome features (found in all 121 Candida species) and accessory genome features (shared or unique functions) ${ }^{65}$. Intra-strain 122 analysis of C. albicans across 24 strains sequenced showed largely conserved metabolic 123 pathways and CAZymes (Extended Figure i-j). In covering KEGG metabolic orthologs, 124 clustering analysis determined a larger number of accessory features of metabolism compared 125 to core characteristics seen in all Candida strains (Fig. 1d, Extended Fig. 1f-h, Method).

\section{Identification of global functional annotation profiles in Candida}

128 We next determined the CAZyme profile by mapping the 49 Candida protein sequences to 129 the dbcan2 database ${ }^{64}$. Doing this allowed us to infer molecular enzyme function ${ }^{66}$. 130 CAZymes are vital enzymes involved in the metabolism of complex carbohydrates. 131 Approximately 205 unique CAZymes were identified in all Candida strains, with various 132 functions (Fig. 1f, Supplementary Table 6, Method). From core Candida genome analysis, 133 annotated enzymes were distributed across 6 active families: providing an assortment of 134 enzymatic functions. The glycoside hydrolase (GH) family showed the highest degree of core 
135 coverage (Extended Fig. 2a), with much of the GH family activity in starch and other storage 136 carbohydrates substrate-converters (Fig. 1g).

137 Additionally, xylan and sugar carbohydrate utilisation were the dominant functions in the 138 accessory genome (Supplementary Table 6). Cell wall composition is a crucial virulence 139 factor and assessing CAZyme components of cell wall substrate converters has been 140 extensively researched ${ }^{67}$. Here, we reveal the presence of pectin lyases, glycan lyases, chitin 141 lyases and mannan lyases (Fig. 1h). Pectin substrate conversion enzyme has been identified 142 as the core feature of Candidas' functional cell wall enzyme, though previously only reported 143 in Candida bodinii ${ }^{68}$ and frequently seen in the fungal plant pathogen, including Aspergillus 144 Pencillium ${ }^{69}$. Alongside $\beta$-glucan, mannan and chitin carbohydrate enzyme profiles, Candida cell wall activity includes pectin enzyme activity (Supplementary Table 7).

In addition, we identified 1,182 Pfam clans from all Candida strains and re-categorised them into 14 functional clans (Supplementary Table 8). Pfam domain annotation indicating genetic information processing, cell machinery, and metabolism was among the most extensive Pfam domains exhibited (Extended fig. 2b). We assessed the diverse functional association of protein domains by analysing core functional clans, and determined similar patterns of dominance for carbohydrate, amino acid and lipid processing-associated domains (Extended Fig. 2c).

\section{The functional and metabolomic activity of clinical AGAu Candida strains.}

156 Next, to better explore and understand the link to metabolism and pathogenesis, we clustered

157 the groups based on invasive nature of particular species, linked to contributing to a high 158 percentage of mortality and candidemia (Fig. 2a). C. albicans, C. glabrata and the emerging invasive species C. auris were grouped together (AGAu cluster). Alternative Candida species termed non-AGAu group include opportunistic species that require virulence factors or a defective immune system to cause disease pathology as well as environmental Candida species. The AGAu cluster contains those Candida species most commonly associated with

163 clinical pathology, contributing to a higher percentage of mortality and candidemia ${ }^{16-18,53-}$ ${ }^{55,70}$. This classification of AGAu is analysed and discussed throughout the rest of this paper.

We compared the CAZyme profile coverage of AGAu and non-AGAu groups (Fig. 2b-c,

167 Supplementary Table S9). The CAZyme GH43_8 (substrate-conversion of $\alpha$-L168 arabinofuranosidase/ $\beta$-xylosidase ${ }^{71}$ ) was significantly enriched in AGAu possibly involved 
169 in the breakdown of complex glucans (Wilcoxon signed-rank test, P-value $<0.05$, Method) ${ }^{72}$.

170 The identification of significant CAZymes in the AGAu cluster showed carbohydrate 171 conversion of xylan (GH43_8), mucin (GH95), cellulose (GH66) and copper oxidase family

172 (AA5) ${ }^{73-75}$. Interestingly, GH66 associated with human oral plaque formation ${ }^{73}$ and AA5 173 enzyme has been reportedly linked to fungal defence ${ }^{76}$. CAZymes seen in non-AGAu 174 species showed carbohydrate-binding module families involved in sugar, polysaccharide and 175 cell wall breakdown, including CBM48.

177 The AGAu species are morphologically diverse, (C. albicans is dimorphic, whilst C. glabrata 178 and C. auris are not), they are all potential pathogens. Therefore, we assessed metabolic 179 pathway enrichment in KO annotations of both AGAu and non-AGAu Candida strains 180 (hypergeometric test and Wilcoxon signed-rank test, p-value $<0.05$, Method). We found 181 functional evidence of pathways present in both clusters, indicating a genetic potential for all 182 Candida strains to undertake similar metabolic trajectories, as no differences were observed 183 between cluster group in pathway analysis (Fig. 3a). All Candida strains notably revealed 184 encoded pathways facilitating carbohydrate catabolism within the system; thus, potential to 185 drive increasing metabolic activity, for example through fructose and mannose metabolism. 186 We identified significant enrichment of amino acid metabolism, including arginine, proline, 187 cysteine and methionine metabolism. We also observed significant levels of fatty acid 188 biosynthesis and glutathione metabolism, which have previously been associated with 189 virulence mechanisms ${ }^{77-80}$.

\section{Metabolomics revealed key metabolic pathways assimilated by AGAu group}

192 To elucidate the metabolic trajectory taking place by each cluster group, we performed 193 metabolomics on a collection of 7 clinical Candida isolates, representing the diverse 194 pathogenic species (Fig. 3b, Method). These clinical samples were previously isolated from 195 patients infections ${ }^{53,81-84}$ and were used to evaluate in vitro the critical metabolic activity 196 predicted by our functional analyses (Extended fig. 3a). These Candida isolates were 197 representative of both AGAu and non-AGAu grouped species. The metabolomics data were 198 used for partial least squares discriminant analysis (PLS-DA), which resulted in distinct 199 cluster separation of significant analyte classes (Extended Fig. 3b-d, Method). The PLS-DA 200 model identified distinct high metabolites features uniquely in AGAu Candida species 201 (Extended Fig. 3e). For example, histidine metabolite production in AGAu species supports 202 this metabolite role in systemic infection and is potential an anti-fungal target ${ }^{85,86}$. We also 
203

204

205

206

207

208

209

210

211

212

213

214

215

216

217

218

219

220

221

222

223

224

225

226

227

228

229

230

231

232

233

234

235

identified choline-derived metabolites (choline, phosphatidyl-choline and lysophosphatidylcholine) as increased in certain AGAu species (Fig. 3c). We identified phosphatidylcholines analyte class as contributing the highest number of features across selected clinical Candida species (Extended Fig. 3d), which has been observed previously in the hypervirulent C. albicans (SC5314) $\operatorname{strain}^{38-41}$. Lastly, spermine and spermidine, were found to be significantly associated with AGAu strains, indicating polyamine metabolism could play a functional role in the increased association with disease pathology of these strains.

\section{Integrative global metabolic map of of AGAu Candida species}

Having identified these pathways in silico, we next determined gene expression levels of essential polyamine (SPE11, SPE3), choline (CKI1, TAZ1) and fatty acid (CEM1) pathways in C. albicans from the AGAu cluster to validate these findings (Extended fig. $4 \mathrm{~d}$, Supplementary Table 10 and Methods). All 5 genes showed expression substantiating the activity of these pathways. Our conclusions of key pathway associations draws importance of nitrogen sources, specifically the metabolism of amino acids, in this process (Fig. 4), although to date, Candida species pathogenesis is better known to be driven by carbon sources $^{22,37,89,90}$. AGAu species exhibited increased levels of metabolites in the choline pathway, polyamine and fatty acid biosynthesis pathways that are primarily propagated through arginine, cysteine and methionine pathways (Extended Fig. 4a-c). Based on integration of computational and experimental data revealed fundamental metabolic pathways applied AGAu species providing a link to the major advantage shown by AGAu species across the human body with considerable contributions to pathogenesis. These important pathways include polyamine, choline and fatty acid biosynthesis. For instance, the polyamine pathway is thought to be involved in Candida cell proliferation, and in turn, causes host cellular dysfunction by modulating acetylation levels of aminopropyl groups and inducing autophagy, thus increasing cell life $\operatorname{span}^{89,91,92}$. We observed fatty acid biosynthesis production with large numbers of metabolites of triglycerides featuring in PLSDA and family have previously been reported to promote germination and virulence of AGAu Candida strains (Extended Fig. 3d) $)^{93,94}$. Further, fatty acid biosynthesis is vital in fungal cell membrane viability, energy storage, signalling, and cell proliferation - all functions critical in pathogenesis ${ }^{95-98}$. 


\section{Discussion}

237 BioFung was used for building metabolic maps of key Candida strains and the database 238 provides the mycology community with a resource allowing them to dive more deeply into 239 all fungal species' metabolic capability based on protein encoding genes. Development of 240 data generation technologies development, tools and database for fungal species is currently 241 in its infancy, despite significant advances in these areas for bacteria and archaea. This 242 database enables detailed mechanistic annotations to optimise our understanding of fungal 243 species. to do this, it uses HMM to provide high specificity for fungal annotation. To do this, 244 it is currently the best database available for KEGG-based functional annotation of fungi 245 (Extended fig. 1b-d and Supplementary Table 2-4).

246 Analysis of annotations allowed us to identify the influential AGAu group of Candida 247 strains, highlighting critical metabolic pathways in these strains. In doing so, we developed 248 increased understanding of the metabolism of these strains through integrating multi-omics 249 and experimental data. BioFung can be used extensively to better understand individual 250 fungal species' metabolic pathways but can be extended to explore metabolic interactions 251 between fungi, other organisms, and within the host-mycobiome.

253 Using this tool in combination with metabolomics validation, the AGAu Candida strains appears to be employing specific activity of amino acid metabolism. This shows a degree of metabolic plasticity indicative of fungi, where secretion of these metabolites associated with these pathways aiding in better adaptability to growth, virulence factor production, hyphae and biofilm formation ${ }^{39,99}$, enabling more effective adaptation to a wide variety of environments and habitats. Amino acid metabolism has been proposed as an alternative energy source in stress responses and an alternative to carbon sources for growth. We demonstrate here that all Candida species have an amino acid pathways to employ metabolic remodelling (Fig. 3a) ${ }^{39,42,89}$. However, integrating metabolomics from strains grown with abundant nutrient source, shows that the AGAu group as opposed to other species retains significantly more active in the production of polyamine and choline metabolites, which requires the use of amino acid metabolism for production (Fig. 4). The AGAu group employs arginine, methionine and cysteine metabolism and more extensive exploration and experimental data needed to understand the causal effect of amino acid metabolism. For

267 instance, we have identified a confirmed target pathway for anti-fungal drugs with 268 glutathione metabolism (GSH), attributed to fungal mitochondrial maintenance, preservation 269 of membrane integrity, regulation of transcription factors in stress response and protection 
270

271

272

273

274

275

276

277

278

279

280

281

282

283

284

285

286

287

288

289

290

291

292

293

294

295

296

297

298

299

300

301

302

303

against reactive oxygen species. Reducing activity of GSH is under investigation as supplementary aid for anti-fungal drugs (azoles and echinocandin) against resistant strains 100-103. This finding verifies that pathway enrichment analysis echoes feasibility in clinical relevance within the host.

Among these metabolites identified are the polyamines, including spermine and spermidine. Polyamines play critical roles in normal cell physiology. Spermine is essential for Candida hyphal formation, playing a pivotal role in Candida invasion ${ }^{104}$. Spermidine, meanwhile, drives genetic modification in fungi by regulating cell cycle and translating the modification of eukaryotic initiation factor (eIF) ${ }^{105-107}$. Excessive polyamines prolong yeast survival via delayed DNA degradation, increasing the likelihood of mutations that could contribute to the development of anti-fungal resistance ${ }^{108,109}$. These mutations are an important consideration, given that C. glabrata and C. auris are heavily associated with rising anti-fungal resistance ${ }^{110-112}$. Polyamines have also been shown to be anti-inflammatory, depending on the microenvironment, potentially explaining the additional benefits of secondary metabolites to Candida species by modulating host immune responses ${ }^{113}$. The use of polyamines is not limited to fungi. Bacteria use polyamines to create and maintain biofilms in order to withstand host defences as well as promoting cancers ${ }^{114-116}$. Viruses use polyamines to promote cell proliferation, thereby promoting their propagation and spread. Intervention in polyamine synthesis has a high degree of potential as a target for antimicrobials. DNA viruses upregulate polyamine synthesis in host during infection and blocking polyamine synthesis is a strategy used in broad-spectrum anti-viral ${ }^{117,118,119}$. These examples along with our findings here indicate that manipulating polyamine secretion from Candida species is a realistic target for therapeutic intervention of associated diseases.

Choline metabolism is a critical function for both microbial and host physiology, as demonstrated by the increase seen in AGAu Candida species' related metabolites. Disruption of phospholipid biosynthesis in fungi can occur through inhibition of phosphatidylcholine synthesis, showing preventing virulence within the systemic mice model ${ }^{87,88,120,121}$. Further, acetylcholine is essential in the formation of the chitin wall characteristic of fungi ${ }^{122,123}$. Along with the bacteriome, Candida species contribute towards host acquisition of choline. As understanding of choline metabolism is in its infancy, further investigation of hostmycobiome interactions is needed, potentially providing insights for repurposing potential therapeutic intervention. For instance, lack of choline in humans drives liver dysfunction due 
304 to the accumulation of lipids within hepatocytes, which can lead to fatty liver diseases and

305 even hepatic liver cancer ${ }^{124-128}$.

306

307 Functional analysis indicates that both the AGAu and non-AGAu groups show a great degree

308 of metabolic plasticity (Fig. 3a) ${ }^{56,59,60,129}$. The only functional difference seen between groups

309 was with CAZymes. The AGAu group presented with GH66, which has previously only

310 been associated with the human oral microbiome and as a potential marker for plaque

311 formation $^{73}$. Given that C. albicans is constituent of oral plaque, this is constituent with

312 clinical data (Fig. 2b-c) ${ }^{130}$. The finding of GH43_8 remains relatively inconclusive in

313 function but was recently detected in bacteria as $\beta$-galactofuranosidase ${ }^{131}$. Although the

314 modes of action for both GH43_8 and AA5 are currently unknown their enrichment in the

315 AGAu group may provide a function-targeted biomarker for Candida infection ${ }^{132,133}$. We also

316 highlight fatty acid biosynthesis pathway in AGAu species with significant levels of

317 triglycerides' production detected (Fig. 3c). Fatty acid synthesis has been identified in

318 Candida species previously, with focus on OLE1, FASI \& FASII genes as key indicators to

319 pathogenesis and virulence ${ }^{77-80}$. This validates the notion of targeting fatty acid biosynthesis

320 pathway within Candida species to disrupt Candida overgrowth in the host.

321

322 Our study has addressed the need for functional data and tools for fungal species by 323 developing the BioFung resource using the KEGG database. This enables detailed 324 mechanistic pathway analysis of fungi. Our integrative analysis of the AGAu group 325 (associated with the disease pathogenesis) highlighted key pathways that potentially increase 326 virulence and have associating effects in the host. We hypothesise that these markers can aid 327 in identifying routes for intervention in invasive infection. We suggest polyamine, choline 328 and fatty acid biosynthesis metabolism as inception for further investigation. The presence of 329 these metabolites from AGAu Candida species potentially directly affects host homeostasis 330 within the mycobiome and adverse effects on humans during infection. As such, the AGAu 331 Candida species' metabolic reprogramming may present a novel method of controlling 332 interaction and infection with these fungi. Finally, we focus on fungal metabolism 333 exploration and distinctively towards amino acid metabolism, playing a more significant role 334 in virulence and pathogenicity. 
337 Code availability

338 BioFung is public open access database that can be downloaded at:

339 https://www.microbiomeatlas.org/downloads.php. The instruction and the pipeline scripts for

340 BioFung can be found at our GitHub repository https://github.com/sysbiomelab/BioFung.

341

342 Acknowledgment

343 This study was supported by Engineering and Physical Sciences Research Council (EPSRC),

344 EP/S001301/1, Biotechnology Biological Sciences Research Council (BBSRC)

345 BB/S016899/1, Science for Life Laboratory, Swedish National Infrastructure for Computing

346 at SNIC through Uppsala Multidisciplinary Centre for Advanced Computational Science

347 (UPPMAX) under projects SNIC 2020-5-222, SNIC 2019/3-226, SNIC 2020/6-153 and

348 King's College London computational infrastructure facility, Rosalind

349 (https://rosalind.kcl.ac.uk) for high performance computing. We thank Professor Bernhard

350 Hube for kindly sending C. parapsilosis strain.

351

352

\section{Author contributions}

353 N.B, D.M. and S.S. conceived the project. N.B. performed sample preparation, 354 metabolomics, gene expression data preparation and extraction protocols for the paper. N.B. 355 developed the pipeline, analysis and made the figures. S.L. advised on design, statistical and 356 functional analysis of the data. A.P.and S.S.N. processed qPCR platform. N.B. wrote and 357 drafted the manuscript. J.N. and M.U. provided critical feedback on the data and manuscript.

358 All authors read, edited and reviewed the manuscript.

359

360

\section{Conflict of Interest}

361 The authors declare no competing financial interests. 


\section{Methods and Materials}

364 Genome sequence collection. Genome sequences of 49 Candida species were collected from

365 NCBI database with version release 45 of Ensemble Fungi (date accessed: April 2019) ${ }^{134,135}$.

366 Supplementary information of sample strain, genome ID, ENA ID, Biosample ID, sequence

367 platform, year of collection, sample location of collection, sample tuple and available

368 biological annotation has been provided in Supplementary Table 1. The quality of the

369 sequence was checked to look at GC content, scaffold and genome size (see Supplementary

370 Figure 1b). Nucleotide sequences used with ANI to determine the phylogenetic relationship 371 and determine differences between strains using Pyani package ${ }^{136}$.

372

373 Construction of fungi-specific functional database (BioFung). Kyoto Encyclopedia of

374 Genes and Genomes (KEGG) database were downloaded for the investigation of all 128 375 fungal species (3GB file size) from eukaryote database (5GB file size) (downloaded on 376 August 2019) ${ }^{137}$. Around 1,210,746 genes, which are annotated with 4,717 KEGG orthology

377 (KO), were selected among 128 fungal species genes. There were 6071 fungal genes missing 378 sequence to place into $\mathrm{KO}$, and $105 \mathrm{KO}$ failed in multi-sequence alignment due to default 379 settings (minimum of 3 genes sequence required). Those genes per each KO were performed 380 multiple-sequence alignment by ClustalW and generated Hidden Markov Model (HMM) 381 profiles using the hmm-build function of HMMER software (Figure 1A for workflow and 382 supplementary 2a for coverage $)^{138,139}$. Our database of fungal-specific HMM models of 4,722 383 KOs was freely shared via Github repository (https://github.com/sysbiomelab/BioFung). 384 Missing KO from fungal species was not added due to missing gene sequences from KEGG, 385 or the low number of sequences per $\mathrm{KO}(<3)$, thereby failed to perform ClustalW alignment 386 (See details in Supplementary Table 4). Quality check was performed by comparing BioFung 387 HMMs to pre-trained HMMS for eukaryotes (euk90 and euk100 version 91.0) from Raven 388 Toolbox ${ }^{57,58,}$ and we observed that BioFung coverage was much higher than both eukaryote 389 profiles (Supplementary figure1c).

\section{Functional annotations of individual fungi species}

392 Fungal KO annotation of each species was performed by HMM scanning of BioFung HMM 393 models by HMMER software. An in-depth exploratory analysis was performed by manually 394 checking KO annotations of individual species deep. Pathway abundance for AGAu and non395 AGAu species was performed using KEGG pathway annotations. Hypergeometric testing of 396 pathways with significance confirmed using Wilcoxon signed-rank test $(<0.05)$. CAZymes 
397 annotations were performed by mapping Candida protein sequences using HMMs of 398 dbCAN2 database ${ }^{66}$. Substrate conversion of CAZyme families was checked based on 399 literature review ${ }^{69,140-146}$.

400 Candida protein sequences to map against Pfam-A families using HMMs, that are fully 401 annotated and curated above a threshold ${ }^{63}$. Pfam clans' annotations were sub-set into a 402 broader annotation based on a reported standard function of protein domains (please see 403 Supplementary Table 8).

404

405 Contrasted functional annotation of Candida species. Presence and absence of microbial 406 annotations, i.e., prevalence, was tested for significance based on condition using Chi407 squared tests and odd ratio. Percentage coverage of each was also tested between AGAu and 408 non- AGAu Candida species. Contrasted functional annotations were checked on individual strains and placed into presence/absence to perform chi-squared for significance $(<0.05)$, and

410 the odds ratio was performed to identify enriched and depleted in AGAu samples. Additional

411 significant functional annotations are seen in AGAu cluster (Supplementary Table 9).

413 Clustering of protein sequences. Core, shared, and unique proteins were identified based on 414 sequence similarity by a clustering approach called UCLUST algorithm ${ }^{65}$. In short, UCLUST 415 algorithm was applied to identify similarity in protein-encoding gene sequences by clustering 416 and unique protein sequences were identified if included in singleton protein clusters. Core 417 proteins were identified if corresponding proteins from all 49 species were included in the 418 same cluster. Shared proteins were selected if they did not belong to unique and core 419 proteins. Protein sequence clusters were selected based on a threshold 0.5 for representative 420 seed sequence, a default threshold in UCLUST software (Extended fig. 1h). Based on 421 definitions of the core, shared, and unique proteins, we were able to determine the core, 422 shared and unique annotations for KO and CAZymes, accordingly.

423

424 Strain growth. 8 strains of Candida species (C. albicans (SC5314), C. dubliniensis (CD36), 425 C. tropicalis (CBS94), C. glabrata (CBS 138), C. auris (47477), C. parapsilosis (73/037), 426 and C. krusei (CBS573). Strains were grown in liquid sabouraud dextrose broth (Thermo 427 Scientific-Oxoid microbiology, UK) ${ }^{147}$. All strains were grown in $50 \mathrm{ml}$ falcon tube in a 428 shaking incubator $95 \mathrm{rpm}$ at the temperature of $25^{\circ} \mathrm{C}$ to encompass all growth rates. 429 Timepoint measurement of growth was taken to measure the exponential and stationary phase 
430 of the optical density of 1 at $600 \mathrm{~nm}$ absorbance (iEMS Ascent absorbance 96-well plate

431 reader).

432

433 Collection and targeted metabolomics on fungal extracellular matrix. Mid-exponential 434 phase indicates bioactive metabolites and time points for the extraction of extracellular 435 metabolites (see Supplementary Figure 1a). 500 $\mu 1$ of extracellular medium, proximity to the 436 pellet was removed from growing fungal cells. Samples were placed through $20 \mu \mathrm{m}$ Whatman 437 filter and snap-frozen in liquid nitrogen. Targeted metabolomics performed using the MxP 438 Quant500 kit (Biocrates, Austria). Partial Least Square - Discriminant Analysis (PLS-DA) 439 was performed on targeted metabolomics of fungal extracellular matrices and media as 440 control, using ropls package ${ }^{148}$. First, PLS-DA was performed to distinguish between 441 Candida samples and control (media). Further, PLS-DA was performed to distinguish 442 between AGAu species and non- AGAu Candida samples. PLS-DA indicated a significant 443 difference between AGAu clusters. Further analysis of metabolite concentrations of targeted 444 metabolomics was normalised, and the Wilcoxon rank-sum test was performed to identify 445 critical metabolites and pathways $(<0.05)$.

\section{Validation experiment}

448 RNA was extracted from 3 biological repeats C. albicans (SC5139) using RNA Qiagen 449 Powersoil kit adapted with bead beating with interval placement on dry ice and additional $450100 \mu 1$ of isopropanol. DNAse cleanup performed using RNA clean-up and concentration kit 451 (NORGEN, Biotek corporation). Primers designed for specific amplification of genes SPE1 452 targeting Ornithine Carboxylase, SPE3 gene for spermidine synthase, CKI1 specific for 453 bifunctional choline kinase/ethanolamine kinase, TAZ1 gene focused on 454 lysophosphatidylcholine acyltransferase) and CEM1 gene target for fatty acid synthase 455 (Supplementary Table 10 for primer information). These primers are specific for C. albicans. 456 Other Candida species only predicted gene ontology-based on C. albicans and 457 Saccharomyces annotation (http:/www.candidagenome.org/cgi-

458 bin/GO/goAnnotation.pl?dbid=CAL0000224407\&seq source=C.\%20auris\%20B8441).

459 Conventional RT-qPCR was performed to identify the expression of these critical pathways 460 for samples, two standard curve analysis with RDN25 which encodes the $25 \mathrm{~s}$ rRNA subunit. 


\section{Figure legends}

463 Figure 1. a, Candida strain characterisation. Coverage of Candida sample population per 464 species available with the categorisation of species profiled. Numbers around the pie chart 465 signify the number of strain representation in each location. (Supplementary Table 4 for more 466 information about strains and extended figure 1a for the global representation of Candida 467 strains). b, Genome-based phylogenetic tree. The phylogenetic tree was constructed based on 468 average nucleotide identity (ANI) between all strains revealing evolutionary differences 469 across strains (colour coordinated) and indicating distinct metabolic capabilities. See 470 Extended Fig. 1d for quality of sequences. c, BioFung database creation workflow. Eukaryote annotation from KEGG database parsed to extract all fungal species. They were genes parsed, sequences extracted and reassembled to KO. The multi-sequence alignment was performed on each KO with all corresponding sequence available. HMM, profile built based on each $\mathrm{KO}$ and assembled to provide a more accurate annotation of fungal species for KO. $\mathrm{d}$,

475 Distribution of Candida species based on sample collection and the framework of protein476 encoded genes analysis of Candida strains. Strains isolated from the various location 477 providing relevant clinical association to host mycobiome and environmental species. * 478 indicates clinical strains used for metabolomics. Functional analysis performed on 49 479 Candida species collected from public repositories. Protein sequences were annotated with 480 Pfam, dbCAN2 and BioFung database for biological information. e, Core and accessory overview of the metabolic pathway across Candida strains. Shared genome feature refers to 6-48 species sharing the function and unique genome features is exhibited by less than 5

483 Candida species denoting accessory functions. f, Clustering of carbohydrate-active enzyme 484 profile (CAZyme). Core, shared genome (6-48 strains), and unique genome ( $<5$ strains) 485 illustrates distribution analysis of functions across all Candida species. g, Analysis of enzyme 486 function of glycoside hydrolase family across all Candida strains. h, Breakdown of cell wall composition of core Candida strains with identification of 49 CAZymes.

Figure 2. a, Contribution of individual Candida species to candidemia and mortality. The impact of each species in AGAu species' grouping is attributed in this study (literature-based) 16-20. b, CAZyme analysis across AGAu and non- AGAu groups. Asterisk connotes statistically significant CAZyme. c, CAZyme enrichment and depletion in AGAu strains.

493 Volcano plot showing statistically significant evidence of a relationship between AGAu 494 strains (chi-squared $<0.05$, in red). Odds ratio applied to distinguish the enriched and depleted 495 effect in invasive strains. 
497 Figure 3. a, Pathway enrichment in 49 Candida strains. Coverage of pathways in AGAu and non-AGAu groups indicating genetic pathway potential (hypergeometric testing and 499 Wilcoxon signed-rank test $<0.05$ ). b, Framework outline of metabolomic analysis of Candida 500 species' metabolism levels of bioactive metabolites in Candida strains exhausted media. 501 Computer simulations were performed for pathway analysis, and statistical approach was 502 applied for candidate metabolites that have a potential influence on the host. c, Significantly 503 enriched metabolites detected in metabolomics in AGAu and non- AGAu groups (Wilcoxon 504 rank-sum test $<0.05)$.

505

506 Figure 4. Global map metabolism in AGAu Candida strains. Enriched pathways observed in 507 functional annotation and further validated by metabolomics with key metabolites markedly 508 significant (Colour in pathway red identify significant metabolites, blue indicate significant 509 pathway enrichment).

510

511 Extended Figure 1. a, Map of Candida species collection. Indication of global representation 512 of samples. b, Sequence quality assessment. Genome size variation, GC content, number of 513 contig and outcome of quality of the sequence. c, Comparison of BioFung to available euk90 514 and euk100 KEGG profiles. Euk90_v91 and euk100_v91 are versions of pre-trained hmm for 515 eukaryote database ${ }^{62}$. Coverage of BioFung was higher than of available hmm. d, Venn 516 diagram displays the overlap of annotation. The overlap between BioFung vs euk90_v91, 517 BioFung vs euk100_v91 and comparison of both eukaryote profiles from all candida gene 518 annotation. BioFung still has an enormous scope of unique KO, and small differences 519 between eukaryote profiles can be observed. e, Validation assessing coverage of the yeast520 specific pathway. We performed pathway analysis of $\mathrm{KO}$ annotations containing yeast521 specific cell pathways. Observation of no yeast specific pathways was found in eukaryote 522 annotation. f, Core percentile coverage of functional annotation. First instance, distribution 523 of KEGG module categories reflect functions seen in most microbiota species. Illustrates the 524 extensive functional capability of Candida within each species. g, Core KEGG metabolism 525 exploration in Candida species. Amino acid, carbohydrate and lipid metabolism contributes 526 the greatest make up of gene function in Candida species. This indicates the contribution of 527 different metabolic potential from Candida. h, Uclust quality assessment by looking at the 528 frequency of cluster numbers at 0.5 cut-offs. The cut-ff was inclusive of signature clusters 529 capture and a broad range of core clusters. i, Intra-strain metabolism analysis of C. albicans 
530 indicating genome differentiation. j, Clustering of 24 C. albicans strains of Carbohydrate

531 Active enzymes demonstrating indistinguishable changes in genome.

532 Extended Figure 2 a, Coverage of CAZyme indicates glycoside hydrolase family is the 533 largest contributor in all Candida species. b, Pfam coverage indicates that metabolic 534 processes, genetic information processing and cellular process \& machinery provide the 535 immense repertoire of protein function in all Candida species. c, Core Pfam metabolic 536 process analysis in Candida species. Carbohydrate, amino acid, and lipid-associated 537 processes are the largest metabolomic protein function in Candida strains.

539 Extended Figure 3 a, Growth curve of strains grown in the laboratory. All the strains were 540 grown, and timepoint measurement of OD1 at $600 \mathrm{~nm}$ was taken to obtain the mid541 exponential phase. b, Targeted metabolomics with PLS-DA analysis differentiating from 542 media. PLS-DA score indicated a precise fitting performance of clustering media distinctly 543 from Candida strains. c, Targeted metabolomics PLS-DA analysis based on AGAu and non544 AGAu species. PLS-DA score plot indicated a fitting and predictive performance (2 latent 545 variables, $R^{2} X=0.501, R^{2} Y=0.984, Q^{2}=0.76$ ). d, Analyte Class component features in PLS546 DA analysis. A significant number of differential components between invasive and non547 invasive strains. e, Heatmap of significant metabolites. Identified from PLS-DA, top 20 548 metabolites differentiated in AGAu and non-AGAu based on Wilcoxon rank-sum test $549(>0.05)$.

551 Extended Figure 4 a, Choline metabolic pathway. Concise choline metabolism and its affect.

552 b, Fatty acid metabolism. Concise fatty acid biosynthesis metabolism and its affect. c, 553 Polyamine metabolism. Concise polyamine metabolism and its affect. d, Quantitative RT554 qPCR validation of pathways with C. albicans. Indicating pathway genes (SPE1, SPE3, CK1, 555 TAZ1, CEM1) compared to their relative expression in Candida albicans, representative of 556 AGAu species cluster. $(\mathrm{n}=3$ biological replicates. Display the relative RNA expression level 557 (Ct value)).

559 Supplementary Table 1: Information on database collection of 49 Candida strains. In-depth 560 data collection of strain retrieval from a database, including strain id, genome id, sample 561 type, origin country, year of collection, annotation availability. 
562 Supplementary Table 2: Fungal species in KEGG database. List of 128 fungal genes in 563 KEGG database, species abbreviation, $\mathrm{T}$ number, protein-encoding genes and genes 564 converted to $\mathrm{KO}$.

565 Supplementary Table 3: The building of BioFung. List of fungal-specific KO available in 566 KEGG database, genes available per $\mathrm{KO}$, missing genes from $\mathrm{KO}$, missing sequences of 567 genes unable to include and failure of multi-sequence alignment in creating BioFung.

568 Supplementary Table 4, Missing information from the KEGG database. List of genes in 569 fungal species had missing sequences and was unable to collate in profile.

570 Supplementary Table 5, AGAu strain categorisation. Based on sample names, grouping, 571 location of sample extraction and reference for strain.

572 Supplementary Table 6, The categorisation of Pfam clans. Categories were based on clan 573 function to the associated processes; note clans can fit into many functional categories.

574 Supplementary Table 7, Significant of functional annotation in enriched or depleted in 575 AGAu group. Some annotations are statistically significant with the odds ratio of falling 576 within the scale of 0 and 1 . They have not been included in the figure but remain relevant. 577 These have been captured in this table for CAZyme, KO and Pfam.

578 Supplementary Table 8, References for Pectin CAZyme assay confirmation. List of 579 publications confirming the function of pectin. Table 9, References for RT-qPCR validation. 580 List of gene selection, primer sequence and accession numbers for gene expression analysis. 


\section{References}

582

583

584

585

586

587

588

589

590

591

592

593

594

595

596

597

598

1. Kam, A. P. \& Xu, J. Diversity of commensal yeasts within and among healthy hosts.

Diagn. Microbiol. Infect. Dis. 43, 19-28 (2002).

2. Bougnoux, M.-E. et al. Multilocus sequence typing reveals intrafamilial transmission and microevolutions of Candida albicans isolates from the human digestive tract. J. Clin.

Microbiol. 44, 1810-1820 (2006).

3. Angebault, C. et al. Candida albicans Is Not Always the Preferential Yeast Colonizing Humans: A Study in Wayampi Amerindians. J. Infect. Dis. 208, 1705-1716 (2013).

4. Moyes, D. L., Richardson, J. P. \& Naglik, J. R. Candida albicans-epithelial interactions and pathogenicity mechanisms: scratching the surface. Virulence 6, 338-346 (2015).

5. Meeting on Global Surveillance of Antimicrobial Resistance Invasive Candida Infections. https://www.who.int/news-room/events/detail/2018/04/24/default-calendar/meetingon-global-surveillance-of-antimicrobial-resistance-invasive-candida-infections.

6. Invasive Fungal Infections: A Creeping Public Health Threat. ASM.org https://www.asm.org/Articles/2018/September/Invasive-Fungal-Infections-A-CreepingPublic-Healt.

7. Fungal Disease Frequency | Gaffi - Global Action Fund for Fungal Infections. https://www.gaffi.org/why/fungal-disease-frequency/.

8. Nett, J. E. Candida auris: An emerging pathogen "incognito"? PLOS Pathog. 15, e1007638 (2019).

9. Lockhart, S. R. et al. Thinking beyond the Common Candida Species: Need for SpeciesLevel Identification of Candida Due to the Emergence of Multidrug-Resistant Candida auris. J. Clin. Microbiol. 55, 3324-3327 (2017). 
605 10. Guinea, J. Global trends in the distribution of Candida species causing candidemia. Clin.

606 Microbiol. Infect. 20, 5-10 (2014).

607 11. Yapar, N. Epidemiology and risk factors for invasive candidiasis. Therapeutics and Clinical

608 Risk Management vol. 10 95-105 https://www.dovepress.com/epidemiology-and-risk-

609 factors-for-invasive-candidiasis-peer-reviewed-article-TCRM (2014).

610 12. Spampinato, C. \& Leonardi, D. Candida Infections, Causes, Targets, and Resistance

611 Mechanisms: Traditional and Alternative Antifungal Agents. BioMed Research

$612 \quad$ International vol. 2013 e204237

613 https://www.hindawi.com/journals/bmri/2013/204237/ (2013).

614 13. Jenkinson, H. F. \& Douglas, L. J. Interactions between Candida Species and Bacteria in

$615 \quad$ Mixed Infections. Polymicrobial Diseases (ASM Press, 2002).

616 14. Pannanusorn, S., Fernandez, V. \& Römling, U. Prevalence of biofilm formation in clinical

617 isolates of Candida species causing bloodstream infection. Mycoses 56, 264-272 (2013).

618 15. Colombo, A. L. et al. Global Distribution and Outcomes for Candida Species Causing

619 Invasive Candidiasis: Results from an International Randomized Double-Blind Study of

620 Caspofungin Versus Amphotericin B for the Treatment of Invasive Candidiasis. Eur. J.

$621 \quad$ Clin. Microbiol. Infect. Dis. 22, 470-474 (2003).

622 16. Pfaller, M. A. et al. Epidemiology and Outcomes of Invasive Candidiasis Due to Non-

623 albicans Species of Candida in 2,496 Patients: Data from the Prospective Antifungal

624 Therapy (PATH) Registry 2004-2008. PLOS ONE 9, e101510 (2014).

625 17. Lindberg, E., Hammarström, H., Ataollahy, N. \& Kondori, N. Species distribution and

626 antifungal drug susceptibilities of yeasts isolated from the blood samples of patients

$627 \quad$ with candidemia. Sci. Rep. 9, 3838 (2019). 
628 18. Adam, R. et al. 378. Candida auris Fungemia: Risk Factors and Outcome. Open Forum

629 Infect. Dis. 5, S147-S147 (2018).

630 19. Almeida, J. N. de et al. Candida haemulonii Complex Species, Brazil, January 2010-

631 March 2015 - Volume 22, Number 3-March 2016 - Emerging Infectious Diseases

632 journal - CDC. doi:10.3201/eid2203.151610.

633 20. Gomez-Lopez, A. et al. Prevalence and Susceptibility Profile of Candida metapsilosis and

634 Candida orthopsilosis: Results from Population-Based Surveillance of Candidemia in

635 Spain. Antimicrob. Agents Chemother. 52, 1506-1509 (2008).

636 21. Aykut, B. et al. The fungal mycobiome promotes pancreatic oncogenesis via activation of

637 MBL. Nature 1-4 (2019) doi:10.1038/s41586-019-1608-2.

638 22. Brown, A. J. P., Brown, G. D., Netea, M. G. \& Gow, N. A. R. Metabolism impacts upon

639 candida immunogenicity and pathogenicity at multiple levels. Trends Microbiol. 22, 614-

$640 \quad 622(2014)$

641 23. Miramón, P. \& Lorenz, M. C. A feast for Candida: Metabolic plasticity confers an edge for

642 virulence. PLOS Pathog. 13, e1006144 (2017).

643 24. Sandai, D. et al. The Evolutionary Rewiring of Ubiquitination Targets Has Reprogrammed

644 the Regulation of Carbon Assimilation in the Pathogenic Yeast Candida albicans. mBio 3,

$645 \quad$ (2012).

646 25. Chew, S. Y. et al. Physiologically Relevant Alternative Carbon Sources Modulate Biofilm

647 Formation, Cell Wall Architecture, and the Stress and Antifungal Resistance of Candida

648 glabrata. Int. J. Mol. Sci. 20, 3172 (2019).

649 26. Sandai, D., Tabana, Y. \& Sandai, R. Carbon Sources Attribute to Pathogenicity in Candida

$650 \quad$ albicans. Candida Albicans (2018) doi:10.5772/intechopen.80211. 
651 27. Tucey, T. M. et al. Glucose Homeostasis Is Important for Immune Cell Viability during

652 Candida Challenge and Host Survival of Systemic Fungal Infection. Cell Metab. 27, 988-

$653 \quad$ 1006.e7 (2018).

654 28. Moyes, D. L. et al. Candidalysin is a fungal peptide toxin critical for mucosal infection.

$655 \quad$ Nature 532, 64-68 (2016).

656 29. Naglik, J. R., Gaffen, S. L. \& Hube, B. Candidalysin: discovery and function in Candida

657 albicans infections. Curr. Opin. Microbiol. 52, 100-109 (2019).

658 30. Rodaki, A. et al. Glucose Promotes Stress Resistance in the Fungal Pathogen Candida

659 albicans. Mol. Biol. Cell 20, 4845-4855 (2009).

660 31. Deacon, J. W. Fungal Biology. (John Wiley \& Sons, 2013).

661 32. Yin, Z. et al. Glucose triggers different global responses in yeast, depending on the

662 strength of the signal, and transiently stabilizes ribosomal protein mRNAs. Mol.

$663 \quad$ Microbiol. 48, 713-724 (2003).

664 33. Childers, D. S. et al. The Rewiring of Ubiquitination Targets in a Pathogenic Yeast

665 Promotes Metabolic Flexibility, Host Colonization and Virulence. PLOS Pathog. 12,

666 e1005566 (2016).

667 34. Lorenz, M. C., Bender, J. A. \& Fink, G. R. Transcriptional Response of Candida albicans

668 upon Internalization by Macrophages. Eukaryot. Cell 3, 1076-1087 (2004).

669 35. Ene, I. V. \& Brown, A. J. P. Integration of Metabolism with Virulence in Candida albicans.

$670 \quad$ Fungal Genomics 349-370 (2014) doi:10.1007/978-3-642-45218-5_14.

671 36. Ene, I. V. et al. Carbon source-induced reprogramming of the cell wall proteome and

672 secretome modulates the adherence and drug resistance of the fungal pathogen

673 Candida albicans. Proteomics 12, 3164-3179 (2012). 
674

675

676

677

678

679

680

681

682

683

684

685

686

687

688

689

690

691

692

693

694

695

696

37. Ene, I. V. et al. Host carbon sources modulate cell wall architecture, drug resistance and virulence in a fungal pathogen. Cell. Microbiol. 14, 1319-1335 (2012).

38. Tudzynski, B. Nitrogen regulation of fungal secondary metabolism in fungi. Front. Microbiol. 5, (2014).

39. Garbe, E. \& Vylkova, S. Role of Amino Acid Metabolism in the Virulence of Human Pathogenic Fungi. Curr. Clin. Microbiol. Rep. 6, 108-119 (2019).

40. Ramachandra, S. et al. Regulatory Networks Controlling Nitrogen Sensing and Uptake in Candida albicans. PLOS ONE 9, (2014).

41. Maidan, M. M., Thevelein, J. M. \& Van Dijck, P. Carbon source induced yeast-to-hypha transition in Candida albicans is dependent on the presence of amino acids and on the G-protein-coupled receptor Gpr1. Biochem. Soc. Trans. 33, 291-293 (2005).

42. Vylkova, S. et al. The Fungal Pathogen Candida albicans Autoinduces Hyphal Morphogenesis by Raising Extracellular pH. mBio 2, (2011).

43. Hudson, D. A. et al. Identification of the dialysable serum inducer of germ-tube formation in Candida albicans. Microbiology, 150, 3041-3049 (2004).

44. Kaur, R., Ma, B. \& Cormack, B. P. A family of glycosylphosphatidylinositol-linked aspartyl proteases is required for virulence of Candida glabrata. Proc. Natl. Acad. Sci. 104, 76287633 (2007).

45. Cheng, S. et al. Profiling of Candida albicans Gene Expression During Intra-abdominal Candidiasis Identifies Biologic Processes Involved in Pathogenesis. J. Infect. Dis. 208, 1529-1537 (2013).

46. Schrevens, S. et al. Methionine is required for CAMP-PKA-mediated morphogenesis and virulence of Candida albicans. Mol. Microbiol. 108, 258-275 (2018). 
697 47. Silao, F. G. S. et al. Mitochondrial proline catabolism activates Ras1/cAMP/PKA-induced

698 filamentation in Candida albicans. (2019) doi:10.1371/journal.pgen.1007976.

699 48. Han, T.-L., Cannon, R. D., Gallo, S. M. \& Villas-Bôas, S. G. A metabolomic study of the

700 effect of Candida albicans glutamate dehydrogenase deletion on growth and

701 morphogenesis. Npj Biofilms Microbiomes 5, 1-14 (2019).

702 49. Naglik, J. R., Challacombe, S. J. \& Hube, B. Candida albicans Secreted Aspartyl

703 Proteinases in Virulence and Pathogenesis. Microbiol. Mol. Biol. Rev. 67, 400-428

704 (2003).

705 50. Richardson, J. P. \& Moyes, D. L. Adaptive immune responses to Candida albicans

706 infection. Virulence 6, 327-337 (2015).

707 51. Mayer, F. L. et al. The Novel Candida albicans Transporter Dur31 Is a Multi-Stage

708 Pathogenicity Factor. PLOS Pathog. 8, e1002592 (2012).

709 52. Global and temporal state of the human gut microbiome in health and disease.

710 https://www.researchsquare.com (2021) doi:10.21203/rs.3.rs-339282/v1.

711 53. Moran, C., Grussemeyer, C. A., Spalding, J. R., Benjamin, D. K. J. \& Reed, S. D. CANDIDA

712 ALBICANS AND NON-ALBICANS BLOODSTREAM INFECTIONS IN ADULT AND PEDIATRIC

713 PATIENTS: COMPARISON OF MORTALITY AND COSTS. Pediatr. Infect. Dis. J. 28, 433-435

714 (2009).

715 54. Hirano, R., Sakamoto, Y., Kudo, K. \& Ohnishi, M. Retrospective analysis of mortality and

$716<$ <em>Candida</em> isolates of 75 patients with candidemia: a single hospital

717 experience. Infection and Drug Resistance vol. 8 199-205

718 https://www.dovepress.com/retrospective-analysis-of-mortality-and-candida-isolates-

719 of-75-patient-peer-reviewed-article-IDR (2015). 
720 55. Horn, D. L. et al. Epidemiology and Outcomes of Candidemia in 2019 Patients: Data from

721 the Prospective Antifungal Therapy Alliance Registry. Clin. Infect. Dis. 48, 1695-1703

722 (2009).

723 56. Nash, A. K. et al. The gut mycobiome of the Human Microbiome Project healthy cohort.

$724 \quad$ Microbiome 5, 153-153 (2017).

725 57. Sam, Q., Chang, M. \& Chai, L. The Fungal Mycobiome and Its Interaction with Gut

726 Bacteria in the Host. Int. J. Mol. Sci. 18, 330-330 (2017).

727 58. Seed, P. C. The human mycobiome. Cold Spring Harb. Perspect. Med. 5, a019810-

$728 \quad$ a019810 (2015).

729 59. Galloway-Peña, J. R. \& Kontoyiannis, D. P. The gut mycobiome: The overlooked

730 constituent of clinical outcomes and treatment complications in patients with cancer

731 and other immunosuppressive conditions. PLOS Pathog. 16, e1008353 (2020).

732 60. Huseyin, C. E., O’Toole, P. W., Cotter, P. D. \& Scanlan, P. D. Forgotten fungi-the gut

733 mycobiome in human health and disease. FEMS Microbiol. Rev. 41, 479-511 (2017).

734 61. Agren, R. et al. The RAVEN Toolbox and Its Use for Generating a Genome-scale

735 Metabolic Model for Penicillium chrysogenum. PLoS Comput. Biol. 9, (2013).

736 62. SysBioChalmers/RAVEN. GitHub https://github.com/SysBioChalmers/RAVEN.

737 63. El-Gebali, S. et al. The Pfam protein families database in 2019. Nucleic Acids Res. 47,

738 D427-D432 (2019).

739 64. dbCAN meta server. http://bcb.unl.edu/dbCAN2/.

740 65. Edgar, R. C. Search and clustering orders of magnitude faster than BLAST. Bioinformatics

$741 \quad 26,2460-2461$ (2010).

742 66. Zhang, H. et al. dbCAN2: a meta server for automated carbohydrate-active enzyme

743 annotation. Nucleic Acids Res. 46, W95-W101 (2018). 
744 67. Zhao, Z., Liu, H., Wang, C. \& Xu, J.-R. Erratum to: Comparative analysis of fungal

745 genomes reveals different plant cell wall degrading capacity in fungi. BMC Genomics 15,

$746 \quad 6(2014)$.

747 68. Nakagawa, T. et al. A Methylotrophic Pathway Participates in Pectin Utilization by

748 Candida boidinii. Appl. Environ. Microbiol. 66, 4253-4257 (2000).

749 69. Barrett, K., Jensen, K., Meyer, A. S., Frisvad, J. C. \& Lange, L. Fungal secretome profile

750 categorization of CAZymes by function and family corresponds to fungal phylogeny and

751 taxonomy: Example Aspergillus and Penicillium. Sci. Rep. 10, 1-12 (2020).

752 70. Sfeir et al. Breakthrough Bloodstream Infections Caused by Echinocandin-Resistant

753 Candida tropicalis: An Emerging Threat to Immunocompromised Patients with

754 Hematological Malignancies. J. Fungi 6, 20-20 (2020).

755 71. CAZy-GH43_8. http://www.cazy.org/GH43_8.html.

756 72. Mewis, K. Functional metagenomic screening for glycoside hydrolases. (University of

757 British Columbia, 2016). doi:10.14288/1.0340610.

758 73. Cantarel, B. L., Lombard, V. \& Henrissat, B. Complex Carbohydrate Utilization by the $759 \quad$ Healthy Human Microbiome. PLOS ONE 7, (2012).

760 74. Katayama, T. et al. Molecular Cloning and Characterization of Bifidobacterium bifidum

761 1,2- $\alpha-I-F u c o s i d a s e(A f c A)$, a Novel Inverting Glycosidase (Glycoside Hydrolase Family 95).

762 J. Bacteriol. 186, 4885-4893 (2004).

763 75. Manzo, N. et al. Carbohydrate-active enzymes from pigmented Bacilli: a genomic

764 approach to assess carbohydrate utilization and degradation. BMC Microbiol. 11, 198

765 (2011). 
766

767

768

769

770

771

772

773

774

775

776

777

778

779

780

781

782

783

784

785

786

787

788

789

76. Ramzi, A. B., Me, M. L. C., Ruslan, U. S., Baharum, S. N. \& Muhammad, N. A. N. Insight into plant cell wall degradation and pathogenesis of Ganoderma boninense via comparative genome analysis. PeerJ 7, e8065 (2019).

77. Nguyen, L. N., Gacser, A. \& Nosanchuk, J. D. The Stearoyl-Coenzyme A Desaturase 1 Is Essential for Virulence and Membrane Stress in Candida parapsilosis through Unsaturated Fatty Acid Production. Infect. Immun. 79, 136-145 (2011).

78. Rodrigues, M. M. Fatty acid synthase 1 in Candida albicans virulence and the in vitro effects of fluconazole, tetracycline in combinatorial therapy. Masters Theses (2012).

79. Southard, S. B. \& Cihlar, R. L. Analysis and expression of the Candida albicans FAS2 gene. Gene 156, 133-138 (1995).

80. Zhao, X. \& Cihlar, R. L. Isolation and sequence of the Candida albicans FAS1 gene. Gene 147, 119-124 (1994).

81. Odds, F. C., Brown, A. J. \& Gow, N. A. Candida albicans genome sequence: a platform for genomics in the absence of genetics. Genome Biol. 5, 230 (2004).

82. Bader, O. et al. Gross Karyotypic and Phenotypic Alterations among Different Progenies of the Candida glabrata CBS138/ATCC2001 Reference Strain. PLOS ONE 7, e52218 (2012).

83. Douglass, A. P. et al. Population genomics shows no distinction between pathogenic Candida krusei and environmental Pichia kudriavzevii: One species, four names. PLoS Pathog. 14, e1007138 (2018).

84. Barchiesi, F. et al. Experimental Induction of Fluconazole Resistance in Candida tropicalis ATCC 750. Antimicrob. Agents Chemother. 44, 1578-1584 (2000).

85. Alcazar-Fuoli, L. Amino acid biosynthetic pathways as antifungal targets for fungal infections. Virulence 7, 376-378 (2016). 
790 86. Jastrzębowska, K. \& Gabriel, I. Inhibitors of amino acids biosynthesis as antifungal

791 agents. Amino Acids 47, 227-249 (2015).

792 87. Tams, R. N. et al. Overproduction of Phospholipids by the Kennedy Pathway Leads to

793 Hypervirulence in Candida albicans. Front. Microbiol. 10, (2019).

794 88. Klig, L. S., Friedli, L. \& Schmid, E. Phospholipid biosynthesis in Candida albicans:

795 regulation by the precursors inositol and choline. J. Bacteriol. 172, 4407-4414 (1990).

796 89. Mayer, F. L., Wilson, D. \& Hube, B. Candida albicans pathogenicity mechanisms.

$797 \quad$ Virulence 4, 119-128 (2013).

798 90. Lorenz, M. C. \& Fink, G. R. The glyoxylate cycle is required for fungal virulence. Nature $799 \quad 412,83-86(2001)$.

800 91. Minois, N., Carmona-Gutierrez, D. \& Madeo, F. Polyamines in aging and disease. Aging $801 \quad 3,716-732(2011)$.

802 92. Valdés-Santiago, L. \& Ruiz-Herrera, J. Stress and polyamine metabolism in fungi. Front.

$803 \quad$ Chem. 1, (2014).

804 93. Noverr, M. C. \& Huffnagle, G. B. Regulation of Candida albicans Morphogenesis by Fatty $805 \quad$ Acid Metabolites. Infect. Immun. 72, 6206-6210 (2004).

806 94. Nguyen, L. N., Trofa, D. \& Nosanchuk, J. D. Fatty Acid Synthase Impacts the Pathobiology 807 of Candida parapsilosis In Vitro and during Mammalian Infection. PLOS ONE 4, e8421 $808 \quad$ (2009).

809 95. Pan, J., Hu, C. \& Yu, J.-H. Lipid Biosynthesis as an Antifungal Target. J. Fungi 4, (2018).

810 96. Gajewski, J., Pavlovic, R., Fischer, M., Boles, E. \& Grininger, M. Engineering fungal de 811 novo fatty acid synthesis for short chain fatty acid production. Nat. Commun. 8, 14650 $812 \quad$ (2017). 
813 97. Tehlivets, O., Scheuringer, K. \& Kohlwein, S. D. Fatty acid synthesis and elongation in

814 yeast. Biochim. Biophys. Acta BBA - Mol. Cell Biol. Lipids 1771, 255-270 (2007).

815 98. Rattray, J. B., Schibeci, A. \& Kidby, D. K. Lipids of yeasts. Bacteriol. Rev. 39, 197-231

$816 \quad$ (1975).

817 99. Desai, J. V. Candida albicans Hyphae: From Growth Initiation to Invasion. J. Fungi 4,

$818 \quad$ (2018).

819 100. Miramón, P. et al. A family of glutathione peroxidases contributes to oxidative stress

820 resistance in Candida albicans. Med. Mycol. 52, 223-239 (2014).

821 101. Liu, Y. et al. Glutathione Reductase Promotes Fungal Clearance and Suppresses

822 Inflammation During Systemic Candida albicans Infection. J. Immunol. 200, 50.1-50.1

$823 \quad$ (2018).

824 102. I, P., Ra, P. \& Mj, P. Glutathione, altruistic metabolite in fungi. Adv. Microb. Physiol.

$825 \quad 49,1-76(2004)$.

826 103. Maras, B. et al. Glutathione Metabolism in Candida albicans Resistant Strains to

$827 \quad$ Fluconazole and Micafungin. PLOS ONE 9, e98387 (2014).

828 104. Dorighetto Cogo, A. J. et al. Spermine modulates fungal morphogenesis and activates

829 plasma membrane $\mathrm{H}^{+}$-ATPase during yeast to hyphae transition. Biol. Open 7,

830 bio029660 (2018).

831 105. Hoyt, M. A. \& Davis, R. H. Polyamines in Fungi. in Biochemistry and Molecular Biology

832 (eds. Brambl, R. \& Marzluf, G. A.) 335-347 (Springer, 2004). doi:10.1007/978-3-662-

833 06064-3_16.

834 106. Tome, M. E. \& Gerner, E. W. Cellular eukaryotic initiation factor 5A content as a

835 mediator of polyamine effects on growth and apoptosis. Biol. Signals 6, 150-156 (1997). 
836

837

838

839

840

841

842

843

844

845

846

847

848

849

850

851

852

853

854

855

856

857

858

107. Belda-Palazón, B. et al. Biochemical quantitation of the elF5A hypusination in Arabidopsis thaliana uncovers ABA-dependent regulation. Front. Plant Sci. 5, (2014).

108. Yu, Y. et al. Position of eukaryotic translation initiation factor elF1A on the 40S ribosomal subunit mapped by directed hydroxyl radical probing. Nucleic Acids Res. 37, 5167-5182 (2009).

109. Chattopadhyay, M. K. et al. Microarray studies on the genes responsive to the addition of spermidine or spermine to a Saccharomyces cerevisiae spermidine synthase mutant. Yeast Chichester Engl. 26, 531-544 (2009).

110. Bhattacharya, S., Sae-Tia, S. \& Fries, B. C. Candidiasis and Mechanisms of Antifungal Resistance. Antibiotics 9, 312 (2020).

111. Hendrickson, J. A., Hu, C., Aitken, S. L. \& Beyda, N. Antifungal Resistance: a Concerning Trend for the Present and Future. Curr. Infect. Dis. Rep. 21, 47 (2019).

112. Silva, S. et al. Candida glabrata, Candida parapsilosis and Candida tropicalis: biology, epidemiology, pathogenicity and antifungal resistance. FEMS Microbiol. Rev. 36, 288305 (2012).

113. Anti-inflammatory effects of spermidine in lipopolysaccharide-stimulated BV2 microglial cells | Journal of Biomedical Science | Full Text. https://jbiomedsci.biomedcentral.com/articles/10.1186/1423-0127-19-31.

114. Li, B. et al. Polyamine-independent growth and biofilm formation, and functional spermidine/spermine $\mathrm{N}$-acetyltransferases in Staphylococcus aureus and Enterococcus faecalis. Mol. Microbiol. 111, 159-175 (2019).

115. Patel, C. N. et al. Polyamines Are Essential for the Formation of Plague Biofilm. J. Bacteriol. 188, 2355-2363 (2006). 
859 116. Villanueva, M. T. Bacterial biofilms may feed colon cancer. Nat. Rev. Cancer 15, 320-

$860 \quad 320(2015)$.

861 117. Li, M. M. H. \& MacDonald, M. R. Polyamines: Small Molecules with a Big Role in

862 Promoting Virus Infection. Cell Host Microbe 20, 123-124 (2016).

863 118. Firpo, M. R. \& Mounce, B. C. Diverse Functions of Polyamines in Virus Infection.

$864 \quad$ Biomolecules 10, 628 (2020).

865 119. Mounce, B. C., Olsen, M. E., Vignuzzi, M. \& Connor, J. H. Polyamines and Their Role in 866 Virus Infection. Microbiol. Mol. Biol. Rev. 81, (2017).

867 120. Wang, J. et al. Phospholipid homeostasis plays an important role in fungal

868 development, fungicide resistance and virulence in Fusarium graminearum.

869 Phytopathol. Res. 1, 16 (2019).

870 121. Chen, Y.-L. et al. Phosphatidylserine synthase and phosphatidylserine decarboxylase 871 are essential for cell wall integrity and virulence in Candida albicans. Mol. Microbiol. 75, $872 \quad 1112-1132(2010)$.

873 122. A, A. \& Sm, K. Acetylcholine Induces Yeast to Hyphal Form Transition in Candida 874 albicans. Fungal Genomics Biol. 08, (2018).

875 123. Markham, P., Robson, G. D., Bainbridge, B. W. \& Trinci, A. P. J. Choline: Its role in the 876 growth of filamentous fungi and the regulation of mycelial morphology. FEMS Microbiol. 877 Rev. 10, 287-300 (1993).

878 124. Mehedint, M. G. \& Zeisel, S. H. Choline's role in maintaining liver function: new 879 evidence for epigenetic mechanisms. Curr. Opin. Clin. Nutr. Metab. Care 16, 339-345 880 (2013). 
881 125. Sherriff, J. L., O’Sullivan, T. A., Properzi, C., Oddo, J.-L. \& Adams, L. A. Choline, Its

882 Potential Role in Nonalcoholic Fatty Liver Disease, and the Case for Human and Bacterial

883 Genes. Adv. Nutr. 7, 5-13 (2016).

884 126. Brown, A. L. et al. Dietary Choline Supplementation Attenuates High-Fat-Diet-

885 Induced Hepatocellular Carcinoma in Mice. J. Nutr. 150, 775-783 (2020).

886 127. Sun, S. et al. Choline and betaine consumption lowers cancer risk: a meta-analysis of 887 epidemiologic studies. Sci. Rep. 6, 35547 (2016).

888 128. Zhou, R. et al. Higher dietary intakes of choline and betaine are associated with a 889 lower risk of primary liver cancer: a case-control study. Sci. Rep. 7, 679 (2017).

890 129. Forbes, J. D., Bernstein, C. N., Tremlett, H., Van Domselaar, G. \& Knox, N. C. A fungal 891 world: Could the gut mycobiome be involved in neurological disease? Front. Microbiol.

$89210,3249-3249$ (2019).

893 130. Zijnge, V. et al. Oral Biofilm Architecture on Natural Teeth. PLOS ONE 5, e9321 894 (2010).

895 131. Mewis, K., Lenfant, N., Lombard, V. \& Henrissat, B. Dividing the Large Glycoside 896 Hydrolase Family 43 into Subfamilies: a Motivation for Detailed Enzyme 897 Characterization. Appl. Environ. Microbiol. 82, 1686-1692 (2016).

898 132. Arfi, Y. et al. Characterization of salt-adapted secreted lignocellulolytic enzymes from 899 the mangrove fungus Pestalotiopsis sp . Nat. Commun. 4, 1810 (2013).

900 133. Kumar, V. et al. Poplar carbohydrate-active enzymes: whole-genome annotation and 901 functional analyses based on RNA expression data. Plant J. 99, 589-609 (2019).

902 134. National Institutes of Health (U.S.). Office of Communications and Public Liaison. NIH 903 research matters.

904 135. Ensembl genome browser 100. https://www.ensembl.org/index.html. 
905 136. Pritchard, L. pyani: pyani provides a package and script for calculation of genome-

906 scale average nucleotide identity.

907 137. KEGG: Kyoto Encyclopedia of Genes and Genomes.

908 https://www.genome.jp/kegg/?sess=ebfe2ad23e021e38540f798c803dd061.

909 138. ClustalW2 < Multiple Sequence Alignment < EMBL-EBI.

$910 \quad$ https://www.ebi.ac.uk/Tools/msa/clustalw2/.

911 139. Eddy, S. R. What is a hidden Markov model? Nat. Biotechnol. 22, 1315-1316 (2004).

912 140. Kovatcheva-Datchary, P. et al. Simplified Intestinal Microbiota to Study Microbe-

913 Diet-Host Interactions in a Mouse Model. Cell Rep. 26, 3772-3783.e6 (2019).

914 141. Zhao, Z., Liu, H., Wang, C. \& Xu, J.-R. Comparative analysis of fungal genomes reveals

915 different plant cell wall degrading capacity in fungi. BMC Genomics 14, 274 (2013).

916 142. Borin, G. P. et al. Comparative Secretome Analysis of Trichoderma reesei and

917 Aspergillus niger during Growth on Sugarcane Biomass. PLOS ONE 10, e0129275 (2015).

918 143. Baroncelli, R. et al. Gene family expansions and contractions are associated with host

919 range in plant pathogens of the genus Colletotrichum. BMC Genomics 17, 555 (2016).

920 144. Looi, H. K. et al. Genomic insight into pathogenicity of dematiaceous fungus

$921 \quad$ Corynespora cassiicola. PeerJ 5, e2841 (2017).

922 145. Wegmann, U. et al. Complete genome of a new Firmicutes species belonging to the

923 dominant human colonic microbiota ('Ruminococcus bicirculans') reveals two

924 chromosomes and a selective capacity to utilize plant glucans. Environ. Microbiol. 16,

$925 \quad 2879-2890$ (2014).

926 146. Geisler-Lee, J. et al. Poplar carbohydrate-active enzymes. Gene identification and expression analyses. Plant Physiol. 140, 946-962 (2006). 
bioRxiv preprint doi: https://doi.org/10.1101/2021.05.24.445215; this version posted May 25, 2021. The copyright holder for this preprint (which

was not certified by peer review) is the author/funder, who has granted bioRxiv a license to display the preprint in perpetuity. It is made available under aCC-BY 4.0 International license.

928 147. Oxoid - Product Detail.

929 http://www.oxoid.com/UK/blue/prod_detail/prod_detail.asp?pr=CM0041.

930 148. Analysis of the Human Adult Urinary Metabolome Variations with Age, Body Mass

931 Index, and Gender by Implementing a Comprehensive Workflow for Univariate and

932 OPLS Statistical Analyses | Journal of Proteome Research.

933 https://pubs.acs.org/doi/full/10.1021/acs.jproteome.5b00354.

$939 B 5$

936

937 

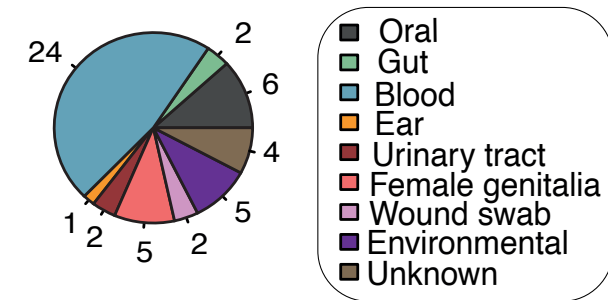

b

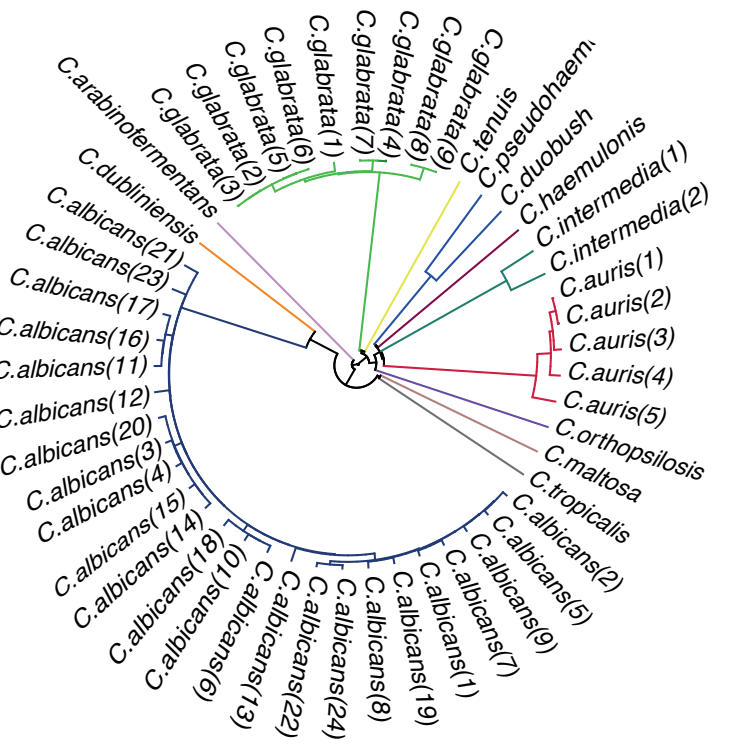

e

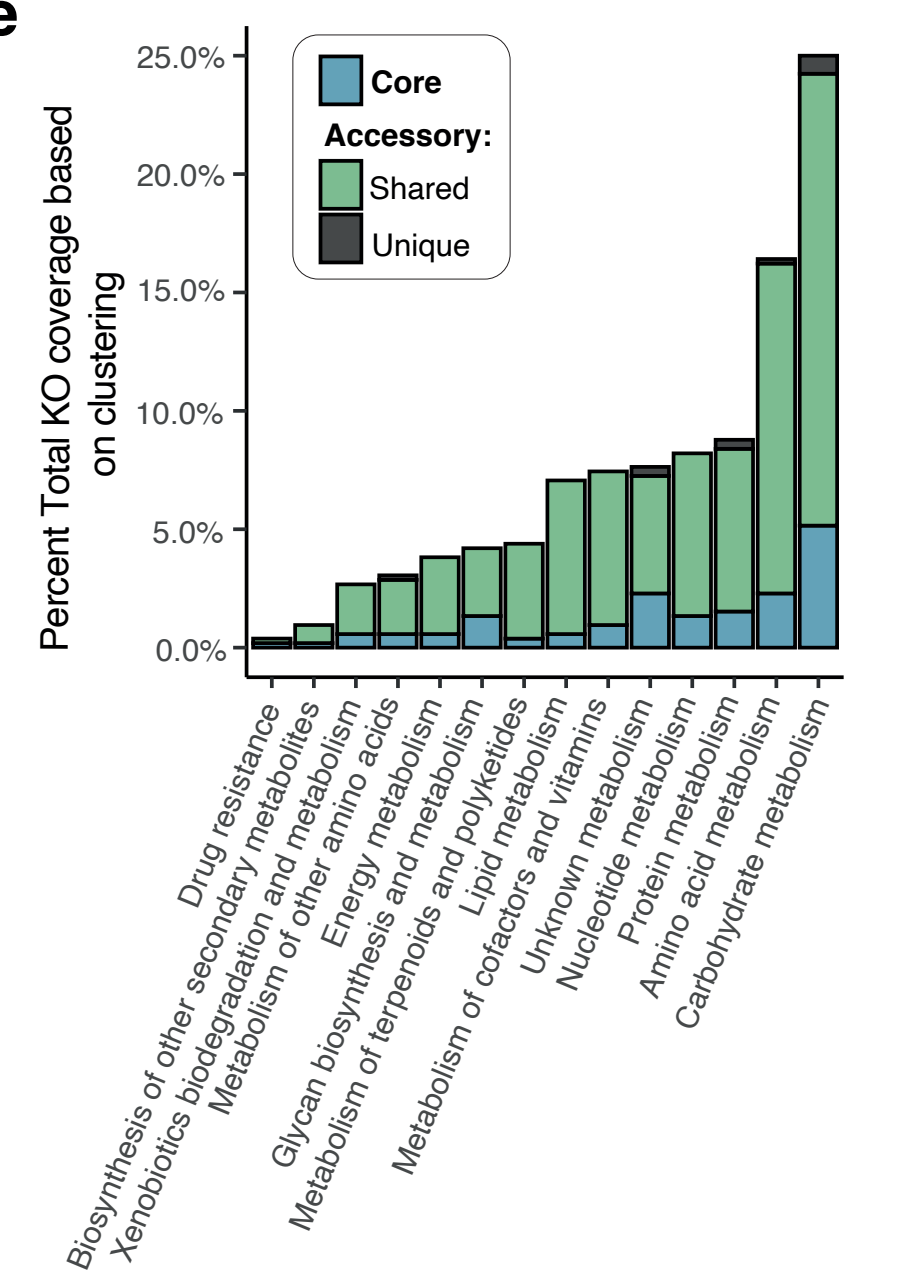

C

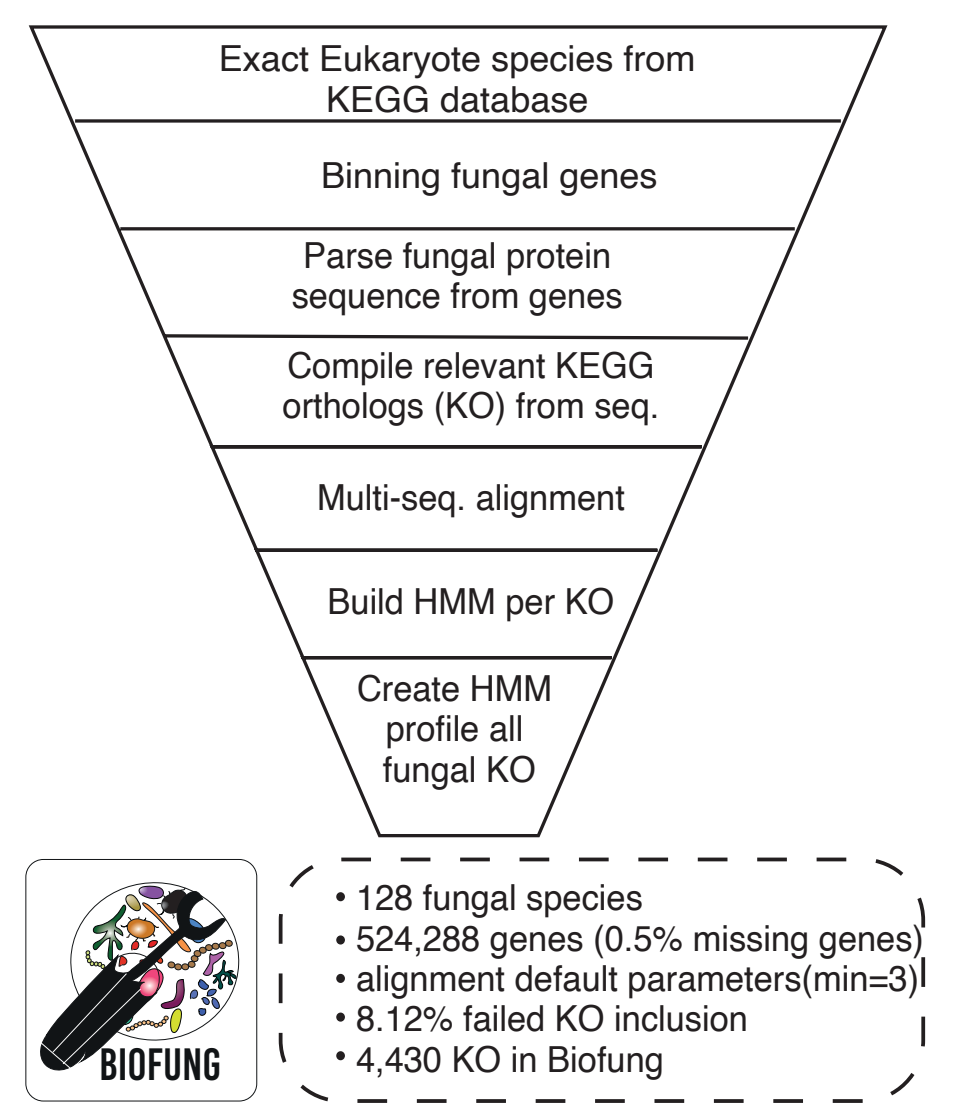

d

Number of samples ○ or $\vec{\circ} \overrightarrow{\mathrm{r}} \sim \tilde{O}$

C. albicans (24)*C.glabrata(9)*C.auris (5)*-

C.dubliensis (1)*-

C.tropicalis (1)*-

C.haemulonis(1) - I

C.orthopsilosis (1) -

C.pseudohaemulonis (1)-

C. duobushaemulonii (1)- I C.krusei *

C.parasilopsis *

C.maltosa(1) -

C.tenuis(1)-

C.intermedia (2)-

C.arabinofermentans (1)-1

Invasive
$\square$ Non-invasive
$\square$ Environmental

$\square$ Non-invasive

(4, $430 \mathrm{KO}$ in Biofung

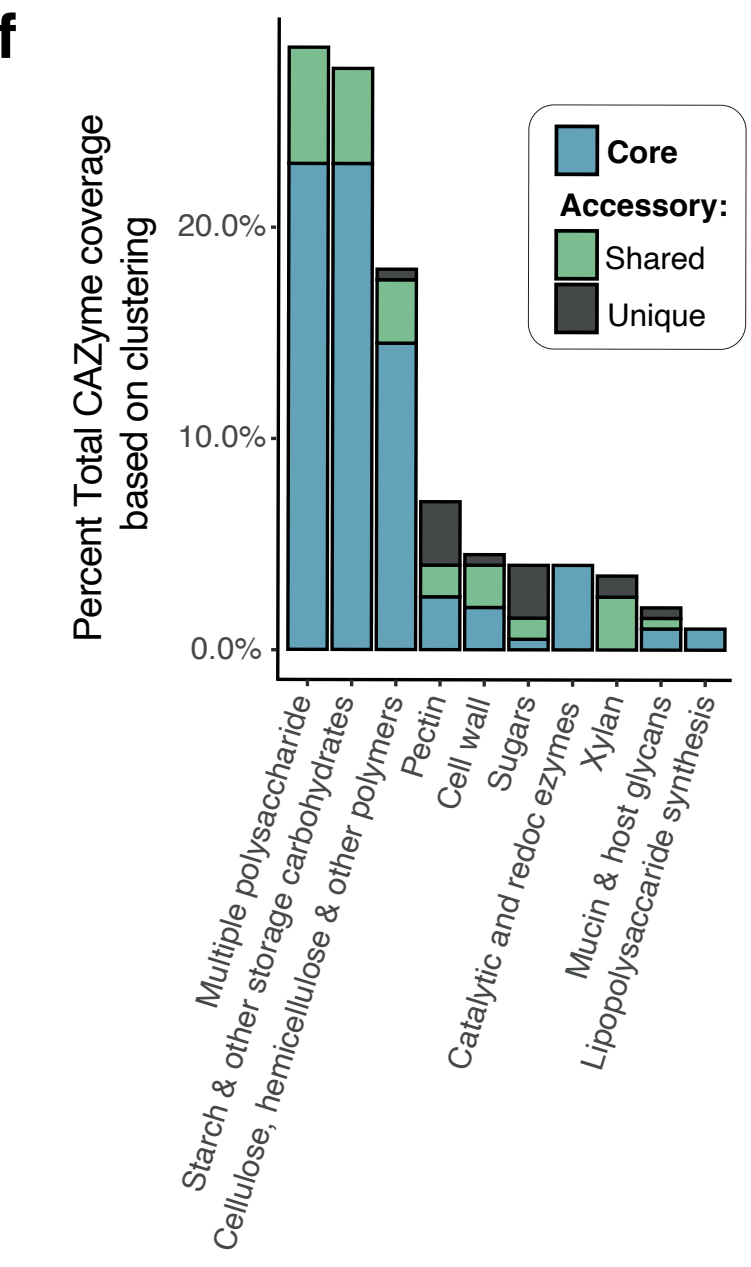

Framework for functional analysis

Collection of 49

Candida species

$$
\Leftrightarrow
$$

Extraction of

protein-encoding gene sequences

Database annotations of Biofung

Pfam

DBCan2

Functional and Cluster analysis

Enrichment and Integration of functional analysis $\cdots \because ?$
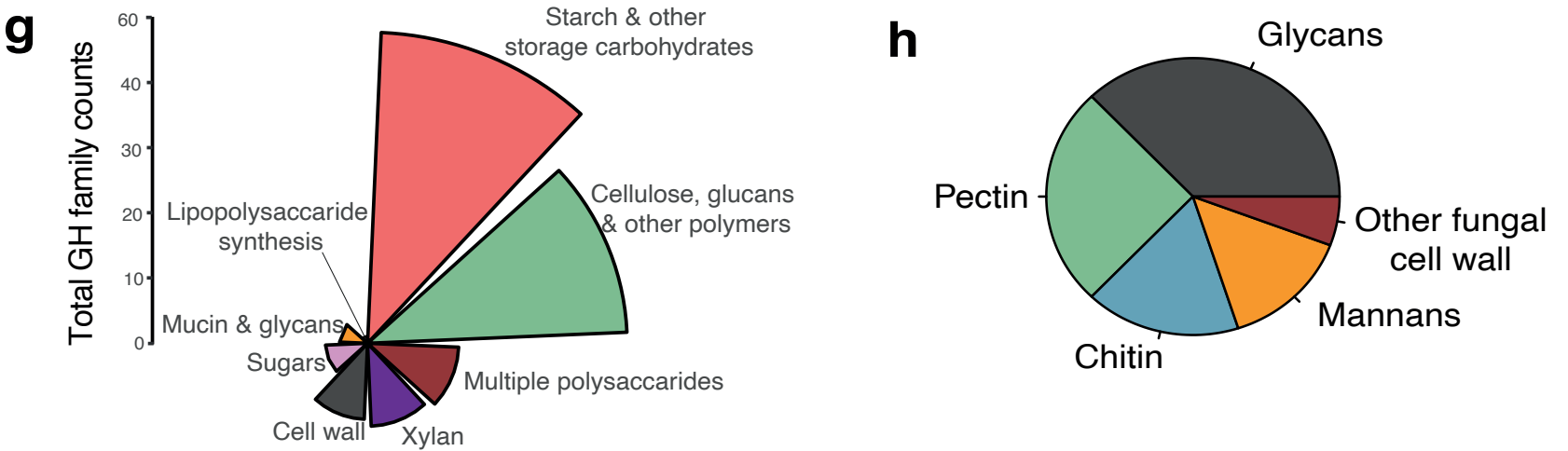


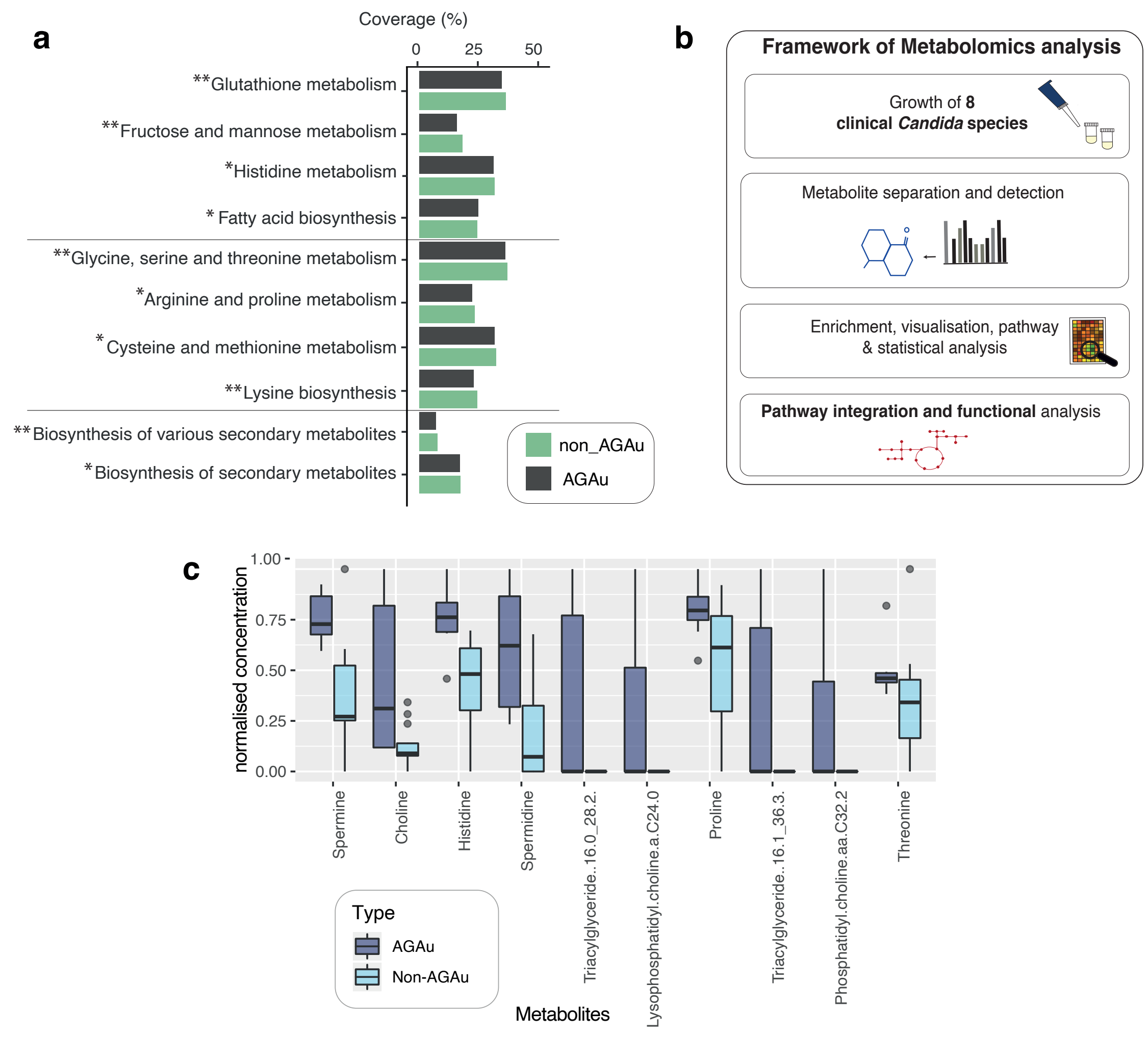


$1.00-$

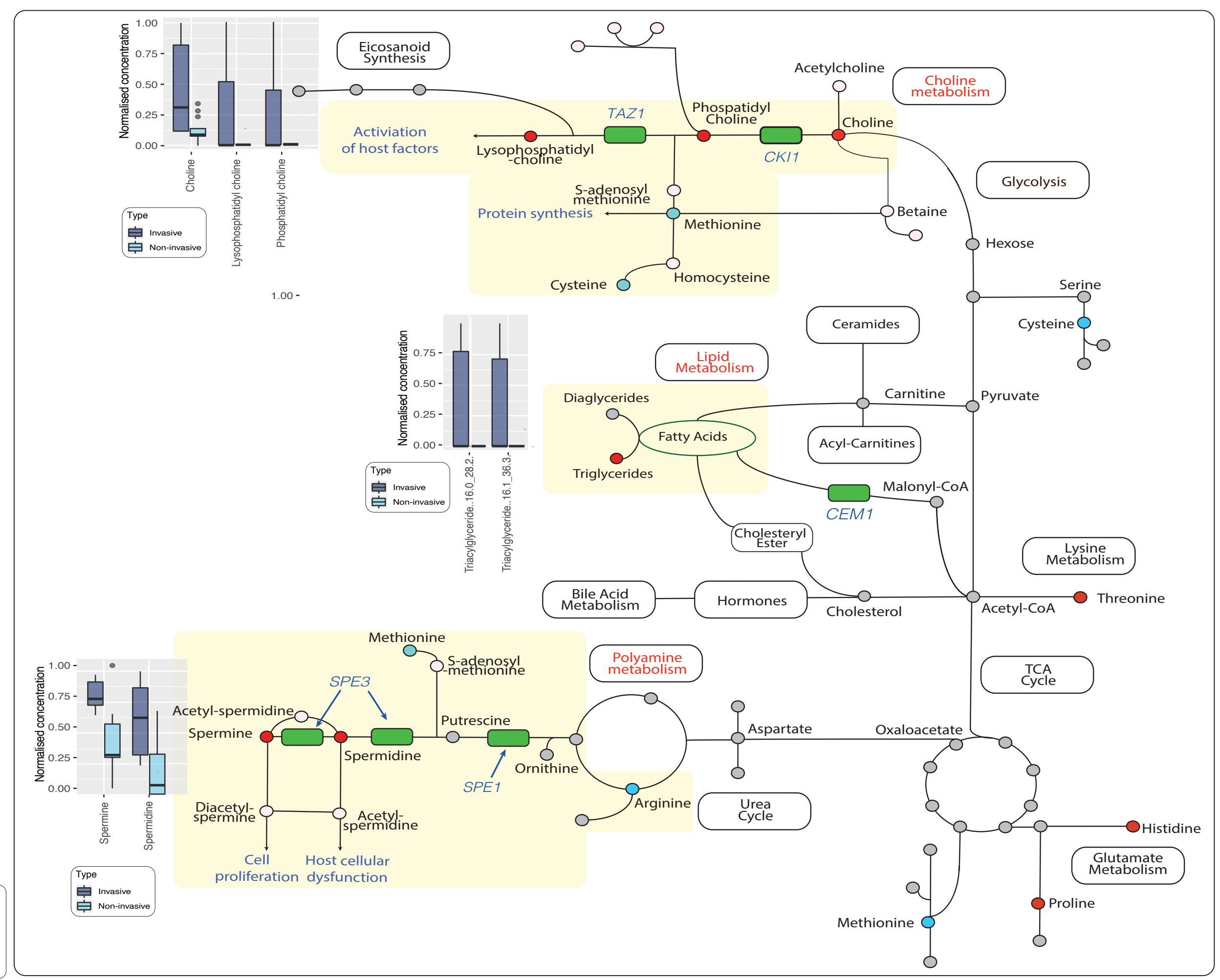




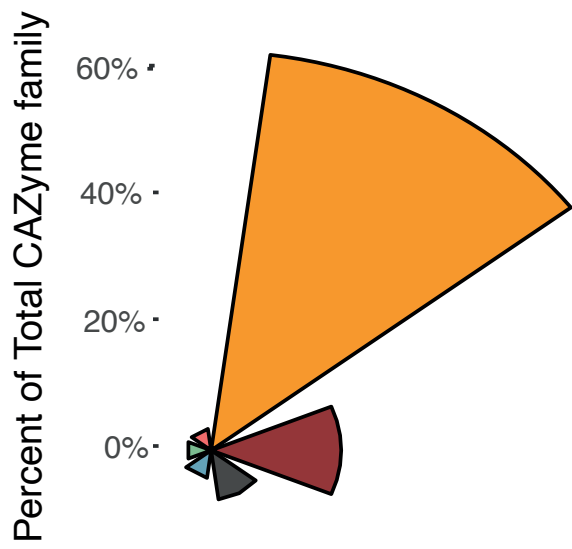

b
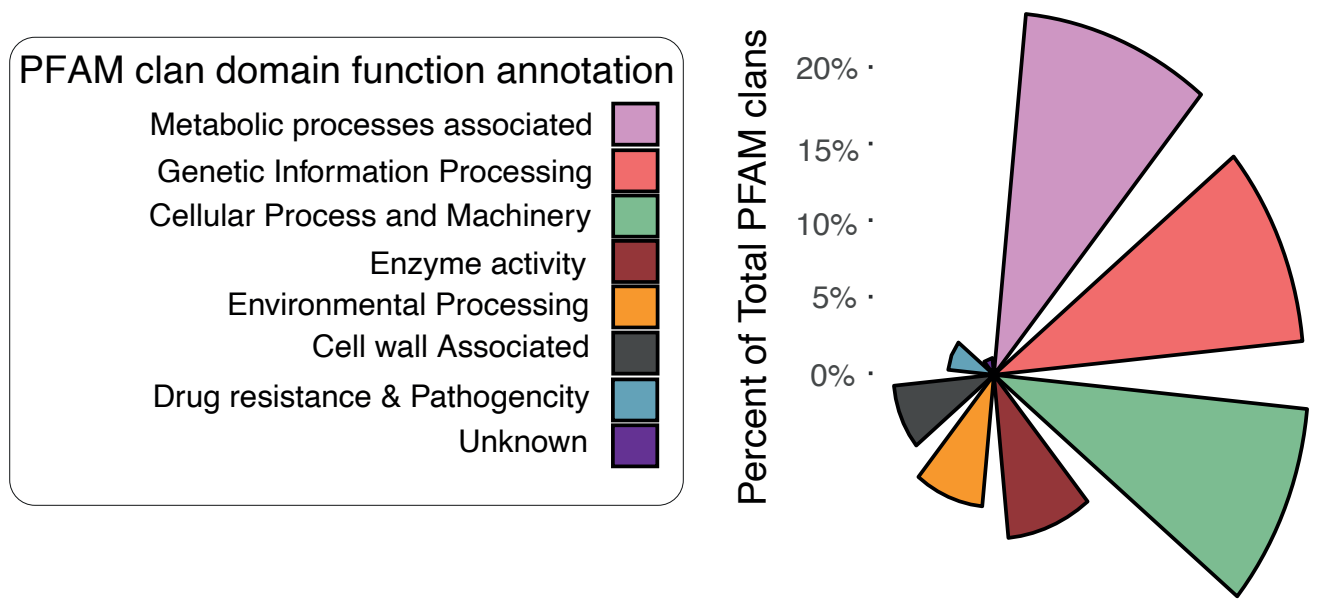

C

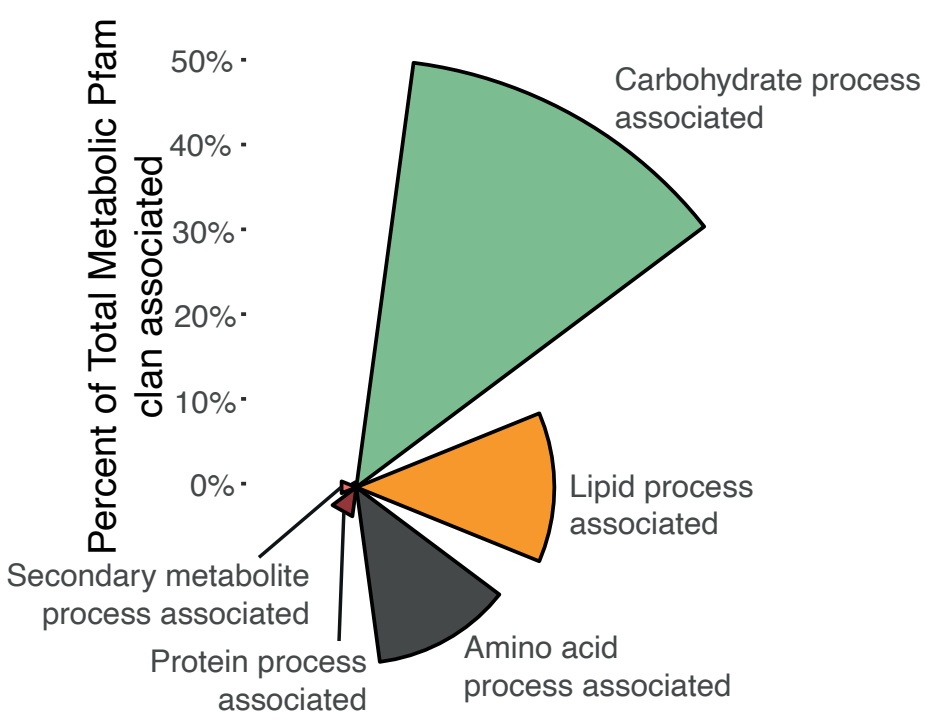


a Logistical growth of Candida strains

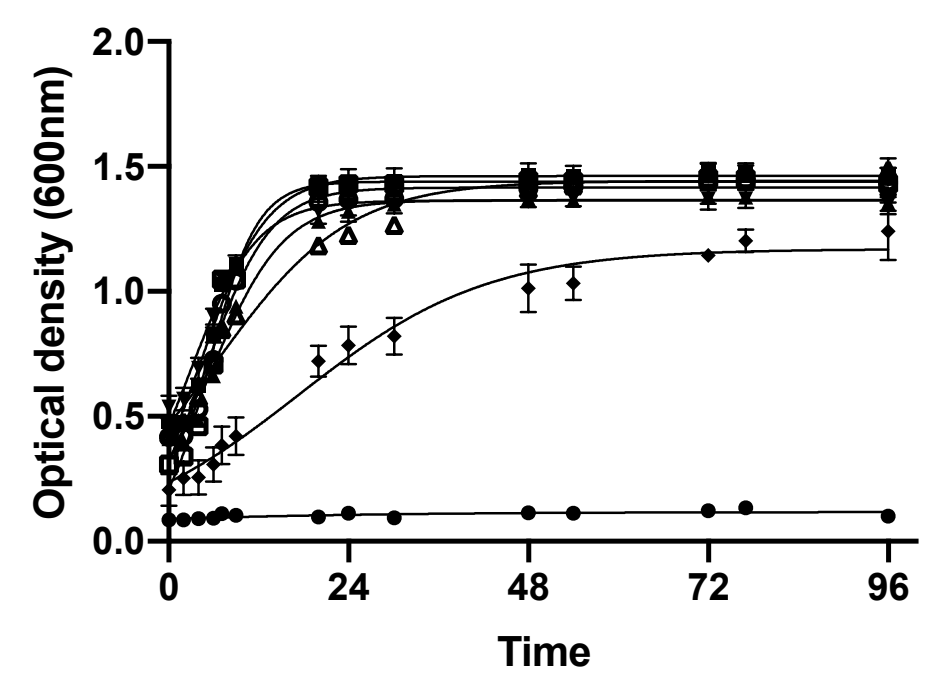

-- blank

$\rightarrow$ C. albicans

$\rightarrow$ C. dubliniensis

$\rightarrow$ C. tropicalis

$\rightarrow$ C. auris

- C. krusei

- C. glabrata

$\triangle$ C. parasilopsis b

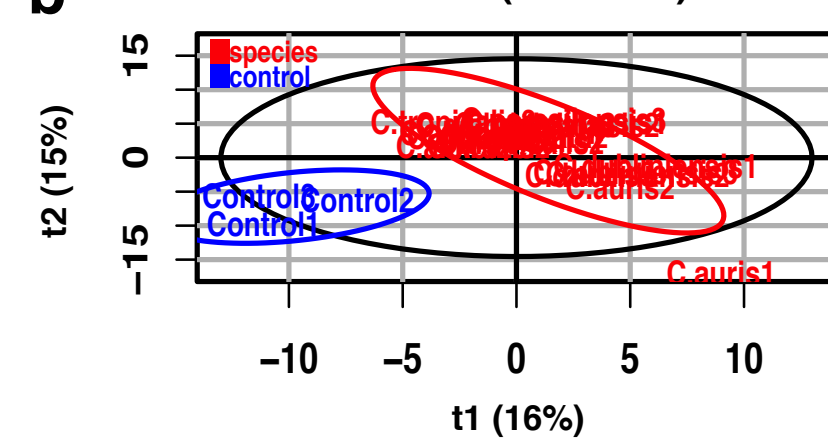

Control vs Species

\begin{tabular}{|l|c|}
\hline PLSDA & R2X \\
\hline Total & 0.524 \\
\hline
\end{tabular}

c

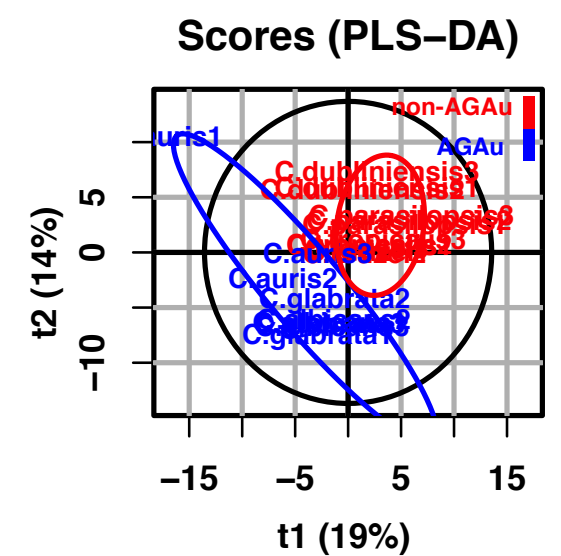

Model overview

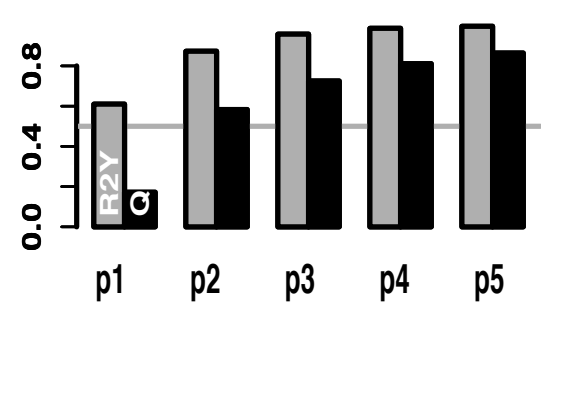

d

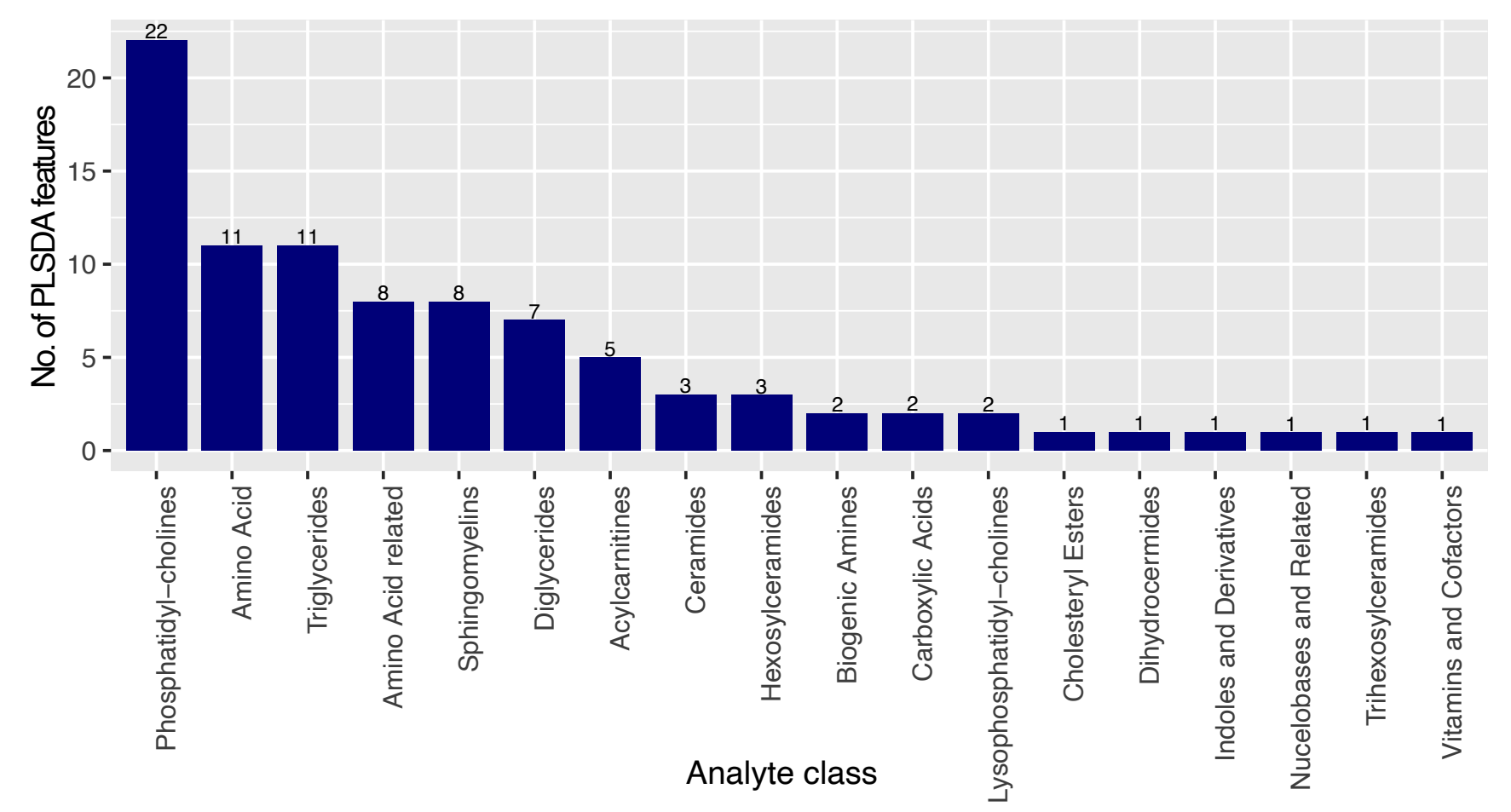

e

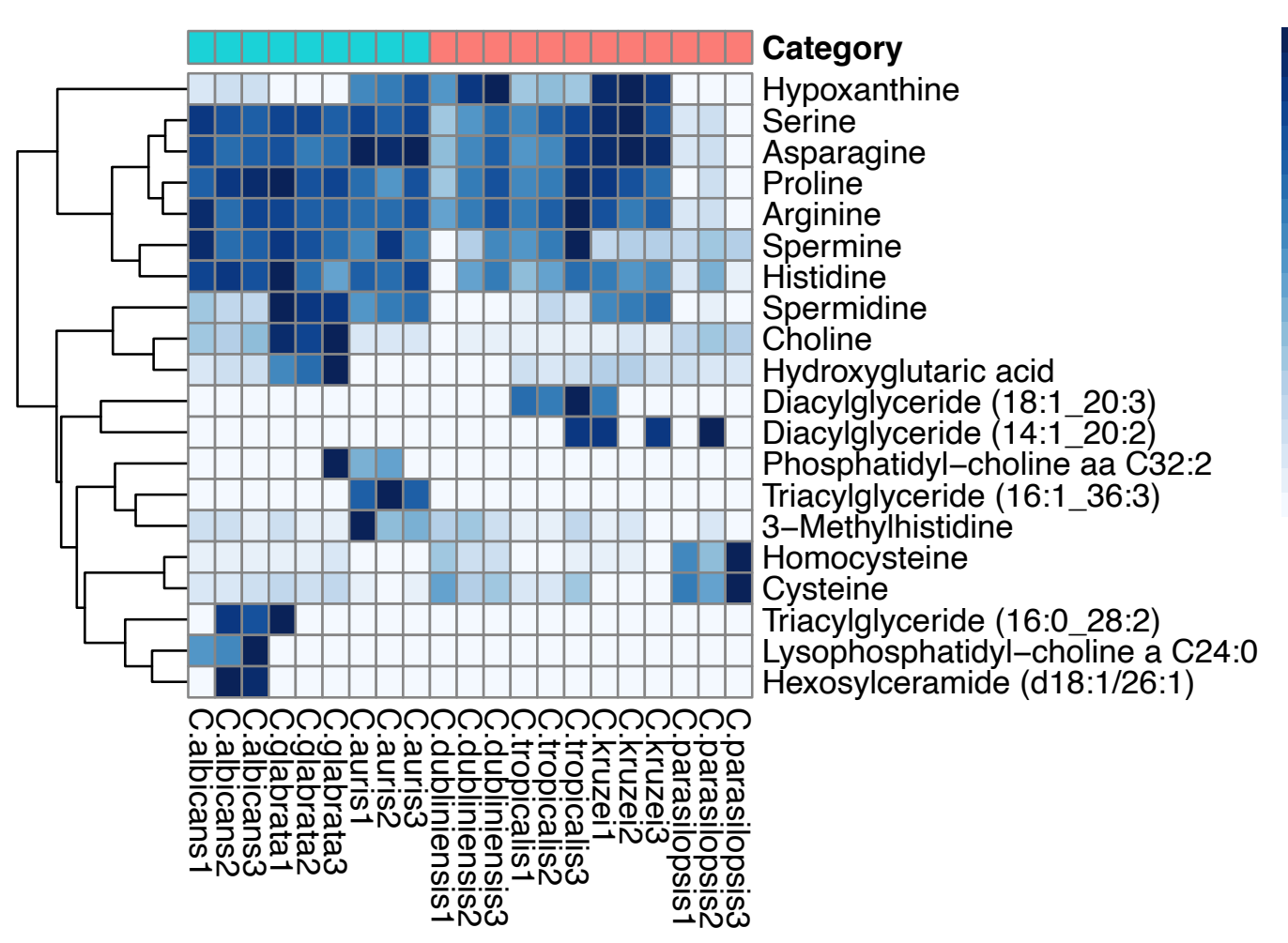

1 Category:

0.8 non-AGAu
nou

0.6

0.4

0.2

0

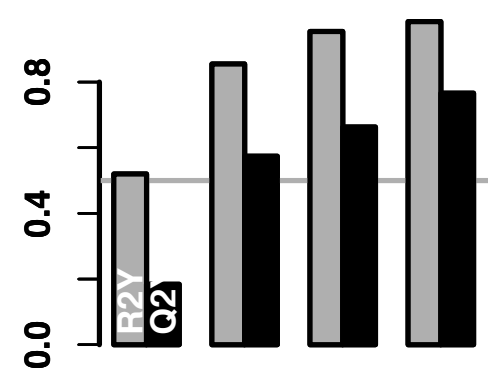

$\begin{array}{llll}\mathrm{p} 1 & \mathrm{p} 2 & \mathrm{p} 3 & \mathrm{p} 4\end{array}$

Invasive vs Non-invasive

\begin{tabular}{|l|c|c|c|c|c|c|c|}
\hline PLSDA & R2X & R2Y(cum) & Q2(cum) & RMSEE & pre & PR2Y & PQ2 \\
\hline Total & 0.501 & 0.984 & 0.766 & 0.071 & 4 & 0.3 & 0.05 \\
\hline
\end{tabular} 


\section{a Choline metabolism}

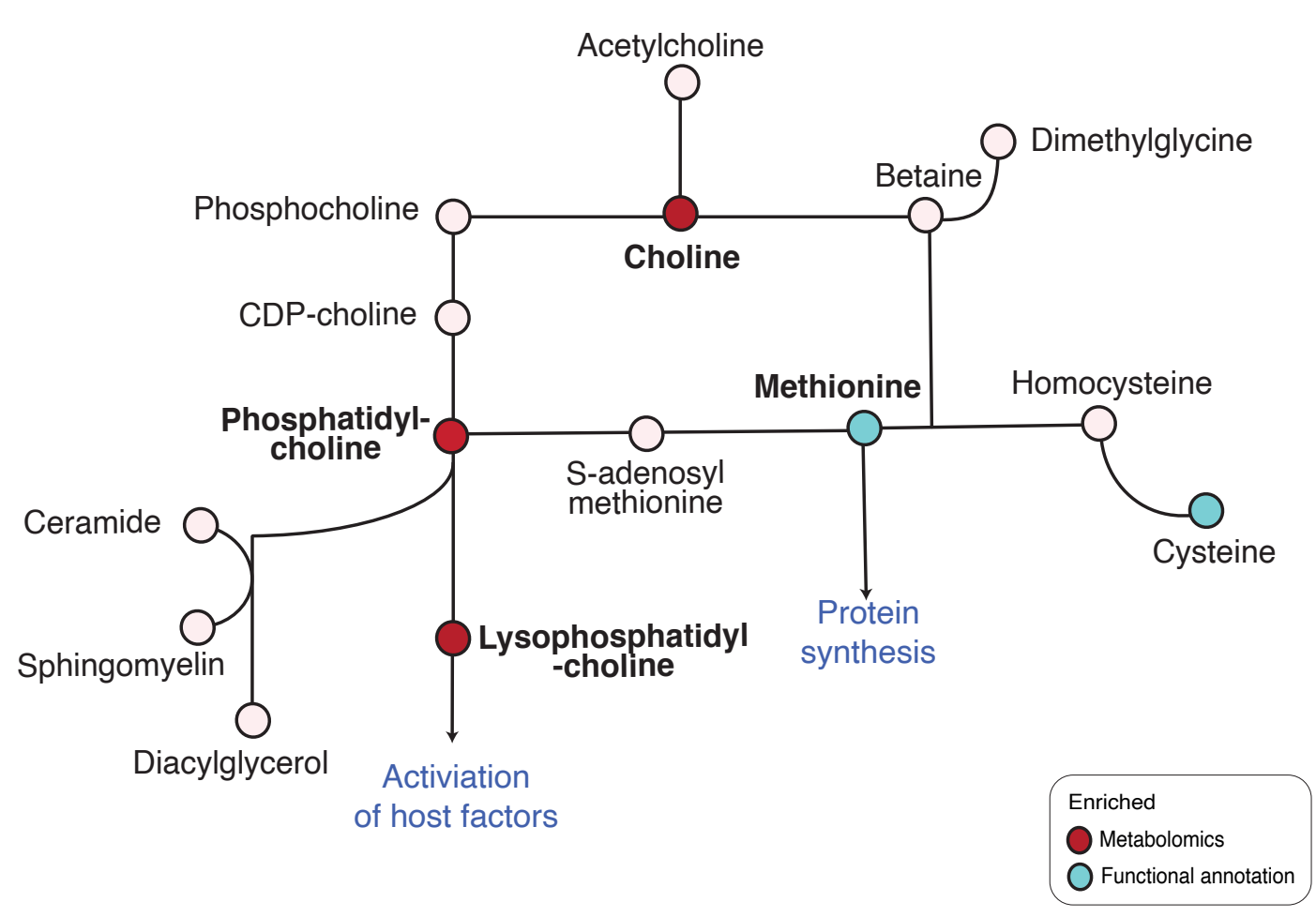

b Fatty acid biosynthesis

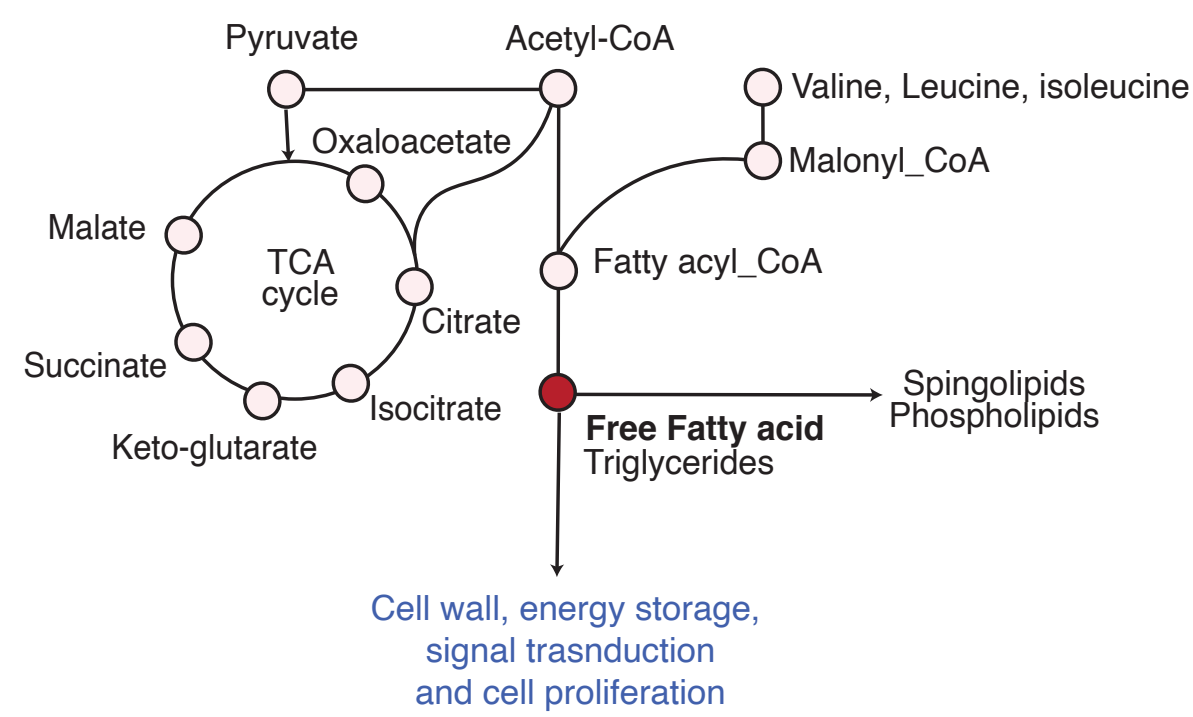

C Polyamine metabolism
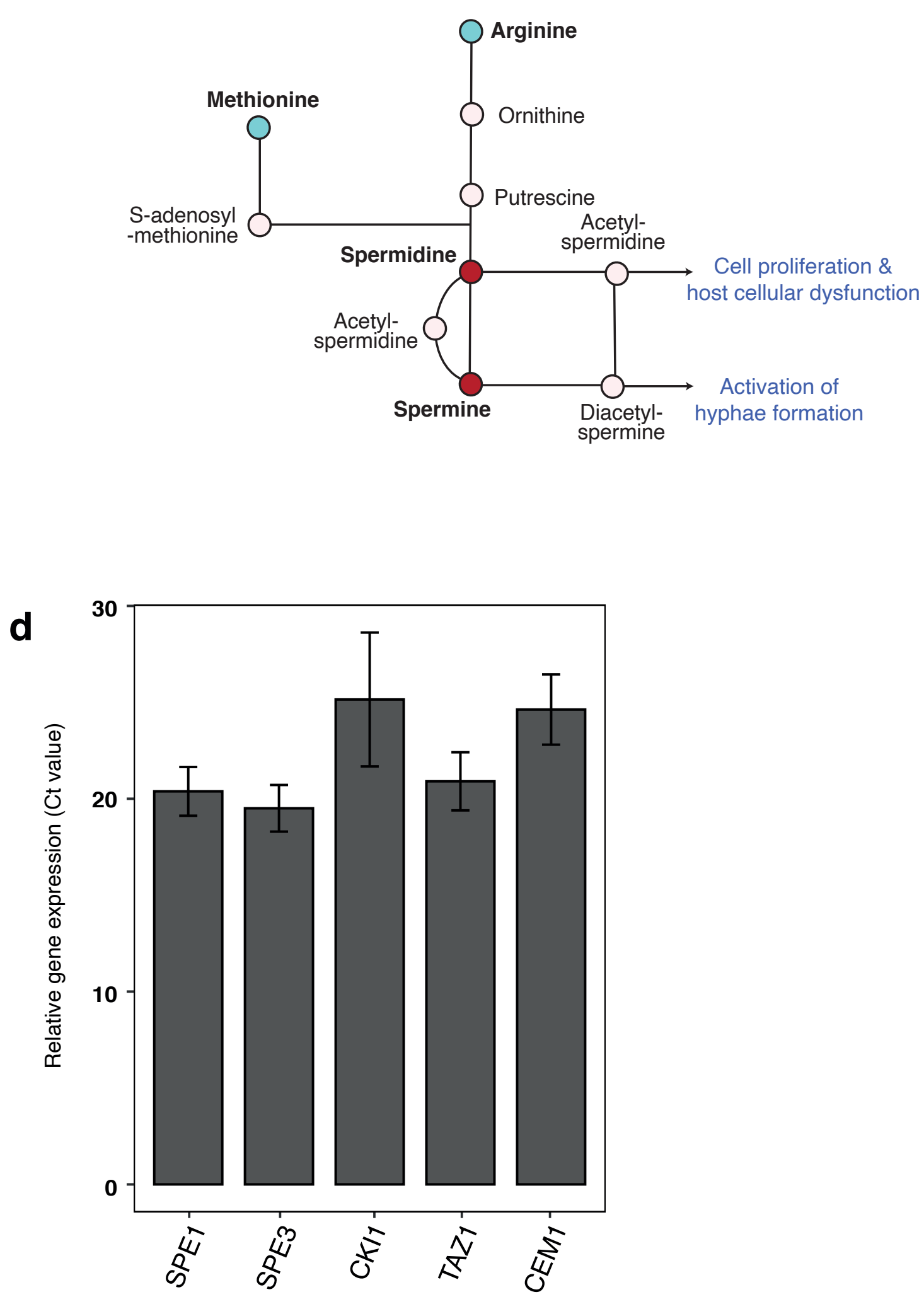

Data source: normalised to number copies of RDN25 ng of cDNA 